\title{
APPLICATIONS OF THE FOURIER-WIENER TRANSFORM TO DIFFERENTIAL EQUATIONS ON INFINITE DIMENSIONAL SPACES. I
}

\author{
BY \\ YUH-JIA LEE ${ }^{1}$
}

\begin{abstract}
Let $(H, i, B)$ be an abstract Wiener space and $p_{t}$ be the Wiener measure on $B$ with variance $t$. Let $[B]$ be the complexification of $B$ and $\varepsilon_{a}$ be the class of exponential type analytic functions defined on $[B]$. We define the Fourier-Wiener $c$-transform for any $f$ in $\varepsilon_{a}$ by$$
\mathscr{F}_{c} f(y)=\int_{B} f(x+i y) p_{c}(d x)
$$

and the inverse transform by $\mathscr{F}_{c}^{-1} f(y)=\mathscr{F}_{c} f(-y)$. Then the inversion formula holds and $\mathscr{F}_{2}$ extends to $L^{2}\left(B, p_{1}\right)$ as a unitary operator. Next, we apply the above transform to investigate the existence, uniqueness and regularity of solutions for Cauchy problems associated with the following two equations: (1) $u_{t}=-\tau^{k} u$, (2) $u_{t t}=-\vartheta^{k} u$; and the elliptic type equation (3) $-\vartheta^{k} u=f(k>1)$, where $\Delta$ is the Laplacian and $\Re u(x)=-\Delta u(x)+(x, D u(x))$.
\end{abstract}

1. Introduction. Gross [6] initiated the theory of differential equations on infinite dimensional abstract Wiener spaces $(H, i, B)$ [5]. $H$ is a given real separable Hilbert space with inner product $\langle$,$\rangle and norm \|=\sqrt{\langle,\rangle} ; B$ is the completion of $H$ with respect to a measurable norm; $i$ is the canonical embedding of $H$ into $B$. In [6], Gross showed that the solution of the Cauchy problem associated with the infinite dimensional heat equation

$$
u_{t}(x, t)=\frac{1}{2} \Delta u(x, t)
$$

can be represented by an integral with respect to the Wiener measure $p_{t}$, where the Laplacian $\Delta$ of a real-valued function $f$ on $B$ is defined as the trace (on $H$ ) of the second Fréchet-derivative $D^{2} f$ of $f$ when the latter exists. Other second order parabolic type differential equations also have this property. For example: (1) Piech [13] represented the solution of the Cauchy problem associated with the second order differential equation with variable coefficients

$$
u_{t}(x, t)=\operatorname{trace}\left[A(x) D^{2} u(x, t)\right]
$$

Received by the editors April 27, 1979.

AMS (MOS) subject classifications (1970). Primary 28A25, 35C10; Secondary 35K30, 35L30, $35 \mathrm{~J} 30$.

Key words and phrases. Abstract Wiener space, Wiener measure, Fourier-Wiener c-transform, exponential type analytic function, equicontinuous semigroup of class $\left(C_{0}\right)$.

'The results are contained in author's doctoral dissertation which was submitted to the Faculty of the Graduate School of State University of New York at Buffalo in partial fulfillment of the requirements for the degree of Ph.D. The author wishes to express his sincere gratitude to Professor M. Ann Piech for her supervision and encouragement during the preparation of this paper.

(c) 1980 American Mathematical Society $0002-9947 / 80 / 0000-0509 / \$ 07.25$ 
as an integral with respect to a measure which is absolutely continuous with respect to the Wiener measure; (2) in [14], Piech investigated the existence and regularity of the Cauchy problem associated with the differential equation $u_{t}(x, t)=$ $-\Re u(x, t)$, where $\Re f(x)=-\Delta f(x)+(x, D f(x))$ (if it exists) and $($,$) denotes the$ natural pairing of $\left(B, B^{*}\right)$. It turns out that the solution can be written as an integral with respect to the transition measure $p_{1-e^{-2 t}}\left(e^{-t} x, d y\right)$.

In the case of differential equations of order greater than two, less work has been done. An approach has been considered recently by Uglanov [19]. There the fundamental solutions of higher order elliptic and parabolic type differential operators of the form $(A \mathscr{D}, \mathscr{D})^{k}(A$ is a constant matrix) are obtained in the sense of generalized measures. However, both localization properties and regularity of solutions are lacking.

In this paper, we shall apply the Fourier-Wiener transform [1] (see 3.1 for definition) and integration by parts [10] to examine Cauchy problems associated with the following types of differential equations:

(1) $u_{t}(x, t)=-\Re^{k} u(x, t)$,

(2) $u_{t t}(x, t)=-\Re^{k} u(x, t)$,

and also the elliptic type differential equation

(3) $-\Re^{k} u(x)=g(x)$

where $k \geqslant 1$.

We owe the definition of the Fourier-Wiener transform to Cameron and Martin [1]. There the Fourier-Wiener transform was defined on a class of mean exponential type analytic functionals on the classical Wiener space $\mathcal{C}$ (i.e. the Banach space of continuous functions on $[0,1]$ vanishing at 0 ) and extended to $L^{2}(\mathcal{C})$ as a unitary operator (see [2]). Later, Segal [16] defined the Fourier-Wiener transform on the class of polynomial cylinder functions on Hilbert space. Hida [7], following [2], developed a more satisfactory Fourier analysis on the dual space of nuclear spaces. However, the natural domain of definition of the Fourier-Wiener transform in [7] is somewhat the same as that of [16]. As far as the applications of the Fourier-Wiener transform are concerned we find that it is desirable to enlarge the (natural) domain of definition of the Fourier-Wiener transform as much as possible (so that it at least also contains a large class of functions other than cylinder functions).

For this purpose, we abstract [1] to define the Fourier-Wiener transform on the class of exponential type analytic functions $\varepsilon_{a}$ on $[B]$-the complexification of $B$. Consequently, if the initial functions are assumed in $\mathcal{E}_{a}$, all the solutions can be represented by series; (1) and (2) have their solutions in $\varepsilon_{a}$, (3) has its solution in $\mathcal{E}_{a}$ provided $g \in \mathcal{E}_{a}$ and $\int_{B} g(x) p_{1}(d x)=0$. The nonhomogeneous cases of $(1)$ and (2) are also considered.

2. Preliminaries. Let $H$ be a real separable Hilbert space with norm $|\cdot|=\sqrt{\langle,\rangle}$. Let $\|\cdot\|$ be a fixed measurable norm on $H$ (for definition, see, for example, [5] or [10]) and $B$ be the completion of $H$ with respect to \|\|$\cdot(H, i, B)$ is called an abstract Wiener space [5], where $i$ is the canonical embedding mapping from $H$ into $B$. If $\mu_{t}(t>0)$ is the Gauss cylinder set measure on $H$ with variance $t$, then $\mu_{t}$ induces a cylinder set measure $\tilde{\mu}_{t}$ on $B$ which in turn extends to a $\sigma$-additive 
measure $p_{t}$ on $(B, \mathscr{B}(B))$, where $\mathscr{B}(B)$ is the $\sigma$-algebra of Borel sets in $B . p_{t}$ is called the Wiener measure with variance $t$ and the following properties hold:

$$
\begin{gathered}
p_{s t}(E)=p_{t}\left(s^{-1 / 2} E\right) \quad \text { for } s>0 ; \quad p_{t}(-E)=p_{t}(E), \\
p_{s} * p_{t}(E)=p_{s+t}(E) .
\end{gathered}
$$

We need the following theorem to prove Theorem 3.7 (integration by parts) which is important in this paper.

Proposition 2.1 (KUo). Let $(H, i, B)$ be an abstract Wiener space. Then there exist another abstract Wiener space $\left(H, i_{0}, B_{0}\right)$ and an increasing sequence of orthogonal projections $\left\{P_{n}\right\}$ converging strongly to the identity in $H$ such that (1) the $B_{0}$-norm is stronger than the $B$-norm (hence $B_{0} \subset B$ ), (2) each $P_{n}$ extends by continuity to $a$ projection $\tilde{P}_{n}$ of $B_{0}$, and (3) $\tilde{P}_{n}$ converges strongly to the identity in $B_{0}$ (w.r.t. $B_{0}$-norm). (For a proof, see [11].)

REMARK 1. It is easy to see that if $p_{t}, \tilde{p}_{t}$ are the Wiener measures on $B$ and $B_{0}$, respectively, then

$$
\int_{B} f(x) p_{t}(d x)=\int_{B_{0}} f(x) \tilde{p}_{t}(d x)
$$

for all nonnegative measurable or integrable functions $f$ on $B$.

Notation. If $A$ is an operator on $H$, we denote by $\|\cdot\|_{t r}$ the trace norm of $A$ and by $\|\cdot\|_{\mathrm{H}-\mathrm{S}}$ the Hilbert Schmidt norm of $A$. We also denote by $\|\cdot\|_{X, Y}$ the operator norm in $L(X, Y)$.

REMARK 2. We note that $B^{*} \subseteq H^{*} \approx H \subseteq B$ and $(x, y)=\langle x, y\rangle$ for $x \in H$, $y \in B^{*}$.

TheOREM 2.2 (Goodman). Let $A$ be a bounded linear operator with range in $B^{*}$ (hence $A \in L\left(B, B^{*}\right)$ ). Then $A$ is a trace class operator on $H$. Moreover,

$$
\|A\|_{\mathrm{tr}} \leqslant \int_{B}\|x\|^{2} p_{1}(d x) \cdot\|A\|_{B, B^{*}}
$$

(We shall see by the next theorem that $\int_{B}\|x\|^{2} p_{1}(d x)<\infty$.)

THEOREM 2.3 (FERNIQUE). There exists $\beta>0$ such that

$$
\int_{B} \exp \left(\beta\|x\|^{2}\right) p_{1}(d x)<\infty .
$$

Corollary 2.4. $\int_{B} \exp (\beta\|x\|) p_{1}(d x)<\infty$ for all $\beta>0$.

REMARK 3. Skorohod showed that the above corollary is true for some $\beta>0$, at approximately the same time as Fernique did in 1970. However, this is obviously an easy consequence of Fernique's result.

For more details about abstract Wiener space we refer to Kuo [11] or the original papers of Gross [3], [4], [5] and Segal [17]. For integration theory on Hilbert space, we refer to Skorohod [19].

We shall denote by $\left([X],\|\cdot\|_{[X]}\right)$ the complexification of a real Banach space $X$, where $[X]=\left\{x_{1}+i x_{2}: x_{1}, x_{2} \in X\right\}$, and $\left\|x_{1}+x_{2}\right\|_{[X]}=\left(\left\|x_{1}\right\|_{X}^{2}+\left\|x_{2}\right\|_{X}^{2}\right)^{1 / 2}$. 
Let $X, Y$ be real Banach spaces, and $[X],[Y]$ be their respective complexifications. We then have

(i) $L(X, Y) \hookrightarrow L([X],[Y])$ by extension and $\|T\|_{X, Y}=\|T\|_{[X],[Y]}$ for $T \in$ $L(X, Y)$.

(ii) If $T \in L([X],[Y])$, then there exists $T_{j}(j=1,2)$ in $L(X, Y)$ such that $T=T_{1}+i T_{2}$ (where we consider $T_{j}$ as an operator in $L([X],[Y])$ in the sense of (i)).

(iii) $\left(\left\|T_{1}\right\|_{X, Y}^{2}+\left\|T_{2}\right\|_{X, Y}^{2}\right)^{1 / 2} \leqslant\|T\|_{[X],[Y]} \leqslant \sqrt{2}\left(\left\|T_{1}\right\|_{X, Y}^{2}+\left\|T_{2}\right\|_{X, Y}^{2}\right)^{1 / 2}$.

Therefore, we may consider $L([X]$, [Y]) as the complexification of $L(X, Y)$.

REMARK 4. If $B$ is a real Banach space, we define $W_{0}=\mathbf{R}$ and $W_{n}=$ $L\left(B, W_{n-1}\right), n=1,2, \ldots$ Then we have $\left[W_{n}\right]=L\left([B],\left[W_{n-1}\right]\right)$.

Now suppose $f$ is an analytic function on some open set $D \subset[B]$ (for more details, we refer to [8], [9]). Then $D^{n} f(x)$ is realized as an operator in $\left[W_{n}\right]$ and $D^{n} f(x)$ is of the form $T_{1}(x)+i T_{2}(x)$ with $T_{1}(x), T_{2}(x) \in W_{n}$ and $T_{1}(x), T_{2}(x)$ extend to $[B]$ in the sense of (i).

3. The Fourier-Wiener transform on abstract Wiener space. Cameron and Martin [1], [2] have defined the Fourier-Wiener transform on the classical Wiener space $\mathcal{C}$. We shall generalize their results to abstract Wiener space. However, the proofs below are quite different from those of [1].

Definition 3.1. Let $(H, i, B)$ be an abstract Wiener space, and $[B]$ the complexification of $B$. If $f$ is a function defined on $[B]$, then for each $y \in[B], x \in B$, $f(x+i y)$ is a function of $x$ defined on $B$. Assuming $f(\cdot+i y) \in L^{1}\left(B, p_{c}\right)$, we define

$$
\left(\mathscr{F}_{c} f\right)(y)=\int_{B} f(x+i y) p_{c}(d x)
$$

and

$$
\left(\mathscr{F}_{c}^{-1} f\right)(y)=\int_{B} f(x-i y) p_{c}(d x) \text { where } c>0 .
$$

We will call $\mathscr{F}_{c} f$ the Fourier-Wiener $c$-transform of $f$, and $\mathscr{F}_{c}^{-1}$ the inverse Fourier-Wiener $c$-transform of $f$. When $c=2, \mathscr{F}_{2} f$ is customarily called the Fourier-Wiener transform of $f$.

Note that $\mathscr{F}_{c} f(y)=\int_{B} f(\sqrt{c x}+i y) p_{1}(d x)$ and $\mathscr{F}_{c}^{-1} f(y)=\mathscr{F}_{c} f(-y)$.

Notation. For notational convenience we use \|\| as the norm for both $B$ and $[B]$. (Since if $x \in B,\|x\|_{B}=\|x\|_{[B]}$.)

Definition 3.2. Denote by $\varepsilon_{a}$ the collection of functions defined on $[B]$ satisfying the following two conditions:

(i) $|f(z)| \leqslant K_{1} \exp \left(K_{2}\|z\|\right)$ for some constants $K_{1}, K_{2}>0$.

(ii) For $x, y \in[B], f(x+\lambda y)$ is an entire function of $\lambda \in \mathbf{C}$.

We denote by $\mathcal{E}_{a}(B)$ those functions which are defined on $B$ and have an extension to $\mathscr{E}_{a}$. (Note that if the extension exists, it is unique.)

REMARK 1. We call $\mathcal{E}_{a}$ the class of exponential type analytic functions. When $B=\mathcal{C}$, the classical Wiener space, $\mathcal{E}_{a}$ contains the space $E_{m}$ of mean exponential type analytic functionals defined and used by Cameron and Martin [1]. We shall 
identify $\varepsilon_{a}(B)$ and $\varepsilon_{a}$ if the domain of definition (i.e. $B$ or $[B]$ ) does not play an important role.

Proposition 3.3. (i) $\mathscr{F}_{c} f$ and $\mathscr{F}_{c}^{-1} f$ exist for $f \in \mathcal{E}_{a}$.

(ii) $\mathscr{F}_{c}\left(\mathcal{E}_{a}\right) \subset \mathcal{E}_{a}$ and similarly, $\mathscr{F}_{c}^{-1}\left(\mathcal{E}_{a}\right) \subset \mathcal{E}_{a}$.

(We shall see later that $\mathscr{F}_{c}\left(\mathcal{E}_{a}\right)=\mathcal{E}_{a}$.)

Proof. (i) As a consequence of Fernique's theorem (see Corollary 2.4) $\exp \left(K_{2}\|x\|\right) \in L^{1}\left(B, p_{c}\right)$. Hence

$$
|f(x+i y)| \leqslant K_{1} \exp \left(K_{2}\|y\|\right) \exp \left(K_{2}\|x\|\right) \in L^{1}\left(B, p_{c}\right)
$$

for every $y \in[B]$ and (i) follows.

(ii) It suffices to show that $\mathscr{F}_{c}\left(\mathcal{E}_{a}\right) \subset \mathcal{E}_{a}$.

In fact,

$$
\begin{aligned}
\left|\mathscr{F}_{c} f(y)\right| & \leqslant \int_{B}|f(x+i y)| p_{c}(d x) \\
& \leqslant K_{1} \int_{B} \exp \left(K_{2}\|x+i y\|\right) p_{c}(d x) \\
& \leqslant K_{1}\left(\int_{B} \exp \left(K_{2}\|x\|\right) p_{c}(d x)\right) \exp \left(K_{2}\|y\|\right) .
\end{aligned}
$$

So 3.2(i) is fulfilled.

Now observe that for any closed curve $\gamma$ in $\mathbf{C}$,

$$
\begin{aligned}
\int_{\gamma}\left(\mathscr{F}_{c} f\right)(x+\lambda y) d \lambda & =\int_{\gamma} \int_{B} f(u+i x+i \lambda y) p_{c}(d u) d \lambda \\
& =\int_{B}\left[\int_{\gamma} f((u+i x)+\lambda(i y)) d \lambda\right] p_{c}(d u) \\
& =\int_{B} 0 \cdot p_{c}(d u)=0 .
\end{aligned}
$$

3.2(ii) follows by Morera's Theorem. Hence, $\mathscr{F}_{c}\left(\mathcal{E}_{a}\right) \subset \mathcal{E}_{a}$.

PROPOSITION 3.4. (a) $\mathcal{E}_{a}$ is a vector space and is closed under multiplication.

If $f \in \mathcal{E}_{a}$, we also have

(b) $f$ is infinitely $F$-differentiable (hence continuous).

(c) $D^{n} f(z) h_{1} \ldots h_{n} \in \mathcal{E}_{a}$, where $h_{1}, \ldots, h_{n} \in[B], n=1,2, \ldots$

(d) $\left\|D^{n} f(z)\right\|_{\left[W_{n}\right]} \leqslant K_{1} \exp \left(n K_{2}\right) \exp \left(K_{2}\|z\|\right)$ for some constants $K_{1}, K_{2}$.

(e) For any $c_{1}>0$, there exist constants $K\left(f, c_{1}\right)$, which depends only on $f$ and $c_{1}$, and $M_{f}$ which depends only on $f$ such that $\left\|D^{n} f(z)\right\|_{\left[w_{n}\right]}<K\left(f, c_{1}\right) M_{f}^{n}$ for all $\|z\| \leqslant$ $c_{1}$.

(f) If $x, y \in[B]$, the Taylor's series (in $y) f(x+y)=\sum_{n=0}^{\infty} D^{n} f(x) y^{n} / n$ ! converges absolutely and uniformly with respect to both $x$ and $y$ on every bounded set in [B].

Proof. By the definition of $\mathcal{E}_{a}$ and the Cauchy formula, the proofs are straightforward.

Moreover, we have 
COROllaRy 3.5. $f \in \mathcal{E}_{a}(B)$ iff

(a) $f$ is infinitely Fréchet-differentiable in $B$, and

(b) for any $c_{1}>0$, there exist constants $K$ and $M$ such that $\left\|D^{n} f(x)\right\|_{w_{n}}<K M^{n}$ for all $\|x\| \leqslant c_{1}$.

The following lemma is a consequence of [10, Theorem 1]. We state it without proof.

LEMMA 3.6. Let $f$ be a continuously Fréchet-differentiable function from $B$ into $B^{*}$. Assume that there is an $r>0$ such that

(i) $\int_{B}\left[\sup _{|h|<r}\|D f(x+h)\|_{B, B^{*}}\right] p_{t}(d x)<\infty$,

(ii) $\int_{B}\|f(x)\|_{B^{*}}^{2} p_{t}(d x)<\infty$.

Then $\int_{B} D f(x) h p_{t}(d x)=t^{-1} \int_{B}\langle x, h\rangle f(x) p_{t}(d x)$ for $h \in H$, where we regard $\langle\cdot, h\rangle$ as a measurable function on $B$ (see, for example, [11]).

Referring to [10, Corollary 1], we have

THEOREM 3.7. Let $f(x)$ be a continuously Fréchet-differentiable function from $B$ into $B^{*}$. If in addition to the assumptions (i) and (ii) of Lemma 3.6, we assume

(iii) $\int_{B}\|D f(x)\|_{\mathrm{tr}} p_{t}(d x)<\infty$, then $\int_{B}(x, f(x)) p_{t}(d x)=t \int_{B} \operatorname{trace}_{H}(D f(x)) p_{t}(d x)$.

Proof. Let $\left(H, i_{0}, B_{0}\right),\left\{P_{n}\right\}$ be the Wiener space and projections respectively in Proposition 2.1 and $\tilde{P}_{n}$ be the extension of $P_{n}$ to $B_{0}$.

By Remark 1 in $\$ 2$, we have

$$
\int_{B}(x, f(x)) p_{t}(d x)=\int_{B_{0}}(x, f(x)) \tilde{p}_{t}(d x),
$$

where (, ) on the right-hand side is the $\left(B_{0}, B_{0}^{*}\right)$ pairing (note that $\left.B_{0}^{*} \supset B^{*}\right)$.

Recall that $\tilde{P}_{n}$ is extended from $P_{n}$ by continuity, so $\tilde{P}_{n}\left(B_{0}\right)$ is the \|\|$_{0}$-closure of $P_{n}(H)$. Since $P_{n}(H)$ is finite dimensional, $\tilde{P}_{n}\left(B_{0}\right)=P_{n}(H)$ (for || and \|\| are equivalent in $\left.P_{n}(H)\right)$.

Let $\left\{e_{j}: j=1,2, \ldots\right\}$ be an orthonormal basis of $H$. Then

$$
\begin{aligned}
\int_{B_{0}}\left(\tilde{P}_{n} x, f(x)\right) \tilde{p}_{t}(d x) & =\int_{B_{0}}\left\langle\tilde{P}_{n} x, f(x)\right\rangle \tilde{p}_{t}(d x) \\
& =\sum_{j=1}^{\infty} \int_{B_{0}}\left\langle e_{j}, f(x)\right\rangle\left\langle\tilde{P}_{n} x, e_{j}\right\rangle \tilde{p}_{t}(d x)
\end{aligned}
$$

(by the dominated convergence theorem)

$$
\begin{aligned}
& =\sum_{j=1}^{\infty} \int_{B_{0}}\left\langle e_{j}, f(x)\right\rangle\left\langle x, P_{n}^{*} e_{j}\right\rangle \tilde{p}_{t}(d x) \\
& =t \sum_{j=1}^{\infty} \int_{B_{0}}\left\langle D f(x) P_{n} e_{j}, e_{j}\right\rangle \tilde{p}_{t}(d x), \quad \text { by Lemma 3.6. }
\end{aligned}
$$

Whence by (iii) and the dominated convergence theorem we have

$$
\int_{B_{0}}\left(\tilde{P}_{n} x, f(x)\right) \tilde{p}_{t}(d x)=t \int_{B_{0}} \operatorname{trace}\left[D f(x) P_{n}\right] \tilde{p}_{t}(d x) .
$$


(Note that if $f$ is Fréchet-differentiable in $B$, then $\left.f\right|_{B_{0}}$ is also Fréchet-differentiable in $B_{0}$ and $D f(x)=D\left(\left.f\right|_{B_{0}}\right)(x)$ for all $x \in B_{0}$.

Recall that $\tilde{P}_{n} \rightarrow I_{B_{0}}$ strongly, we have $\left(\tilde{P}_{n} x, f(x)\right) \rightarrow(x, f(x))$. Also, since $P_{n} \rightarrow$ $I_{H}$ strongly, we have trace $\left[D f(x) P_{n}\right] \rightarrow \operatorname{trace}[D f(x)]$. Moreover,

$$
\left|\left(\tilde{P}_{n} x, f(x)\right)\right|<\|f(x)\|_{B_{0}^{*}}\|x\|_{B_{0}}<\text { const }\|f(x)\|_{B^{*}}\|x\|_{B_{0}} \in L^{1}\left(\tilde{p}_{1}\right),
$$

by assumption (ii), §2, Remark 1 and Hölder's inequality. Besides, $\mid$ trace $D f(x) P_{n} \mid$ $<\|D f(x)\|_{\text {tr. }}$

Therefore by the dominated convergence theorem

$$
\begin{aligned}
\int_{B}(x, f(x)) p_{t}(d x) & =\lim _{n \rightarrow \infty} \int_{B_{0}}\left\langle\tilde{P}_{n} x, f(x)\right\rangle \tilde{p}_{t}(d x) \\
& =\lim _{n \rightarrow \infty} t \int_{B_{0}} \operatorname{trace}_{H}\left[D f(x) P_{n}\right] \tilde{p}_{t}(d x) \\
& =t \int_{B_{0}} \operatorname{trace}_{H}[D f(x)] \tilde{p}_{t}(d x) \\
& =t \int_{B} \operatorname{trace}_{H}[D f(x)] p_{t}(d x) .
\end{aligned}
$$

REMARK 2. It is not hard to see that if $g \in \mathcal{E}_{a}(B)$, then $D g: B \rightarrow B^{*}$ satisfies (i), (ii) and (iii) of Theorem 3.7. In fact (ii) follows by 3.2(1) and Corollary 2.4, and (iii) follows by Goodman's theorem (2.2). For (i), we let $r=1$. Then

$$
\begin{aligned}
\sup _{0<|h|<1}\left\|D^{2} g(x+h)\right\|_{B, B^{*}} & <\sup _{0<|h|<1} c_{g} \exp \left(2 c_{g}^{\prime}+c_{g}^{\prime}\|x+h\|\right) \\
& <c_{g} \exp \left(\left(2+c_{1}\right) c_{g}^{\prime}\right) \exp \left(c_{g}^{\prime}\|x\|\right) \in L^{1}\left(p_{t}\right),
\end{aligned}
$$

where $c_{1}$ is the constant such that $\|h\|<c_{1}|h|$.

LEMMA 3.8. Let $T$ be a symmetric linear operator in $\left[W_{n}\right]$. Then for $x, y \in B$,

$$
\int_{B} \int_{B} T(x+i y)^{n} p_{c}(d x) p_{c}(d y)=0 \text { for } n=1,2, \ldots
$$

Proof. For $n=1$,

$$
\begin{aligned}
\int_{B} \int_{B} T(x+i y) p_{c}(d x) p_{c}(d y) & =\int_{B} \int_{B} T(-x-i y) p_{c}(d x) p_{c}(d y) \\
& =-\int_{B} \int_{B} T(x+i y) p_{c}(d x) p_{c}(d y),
\end{aligned}
$$

since $p_{c}$ is even; hence $\int_{B} \int_{B} T(x+i y) p_{c}(d x) p_{c}(d y)=0$.

For $n=2$,

$$
\begin{aligned}
\int_{B} \int_{B} T(x+i y)^{2} p_{c}(d x) p_{c}(d y) & =\int_{B} \int_{B}\left(T x^{2}-T y^{2}\right) p_{c}(d x) p_{c}(d y) \\
& \quad\left(\text { since } \int_{B} T x y p_{c}(d x)=0\right) \\
& =\int_{B} \int_{B} T x^{2} p_{c}(d x)-\int_{B} \int_{B} T y^{2} p_{c}(d y)=0 .
\end{aligned}
$$


For $n>2$, we write

$$
\begin{aligned}
\int_{B} \int_{B} T(x+i y)^{n} p_{c}(d x) p_{c}(d y)= & \int_{B} \int_{B}\left(x, T(x+i y)^{n-1}\right) p_{c}(d x) p_{c}(d y) \\
& +i \int_{B} \int_{B}\left(y, T(x+i y)^{n-1}\right) p_{c}(d y) p_{c}(d x) .
\end{aligned}
$$

For the first term, we let $f(x)=T(x+i y)^{n-1}$. For the second term, we let $g(y)=T(x+i y)^{n-1}$. Then by Theorem 3.7, we get

$$
\begin{aligned}
(* *)= & c(n-1) \int_{B} \int_{B} \operatorname{trace}_{H} T(x+i y)^{n-2} p_{c}(d x) p_{c}(d y) \\
& -c(n-1) \int_{B} \int_{B} \operatorname{trace}_{H} T(x+i y)^{n-2} p_{c}(d y) p_{c}(d x)=0,
\end{aligned}
$$

where $D f(x)=(n-1) T(x+i y)^{n-2}$ and $D g(y)=i(n-1) T(x+i y)^{n-2}$ are interpreted as operators of the form $A_{1}+i A_{2}$ with $A_{1}$ and $A_{2}$ in $L\left(B, B^{*}\right)$, whence trace $_{H}\left(A_{1}+i A_{2}\right)=$ trace $_{H} A_{1}+i$ trace $_{H} A_{2}$ makes sense.

THEOREM 3.9. For $f \in \mathcal{E}_{a}$, we have

$$
\mathscr{F}_{c}^{-1} \mathscr{F}_{c} f(z)=f(z), \quad z \in[B]
$$

Proof. (Step 1) We claim that (2) holds for $f(z)=T z^{n}, n=1,2, \ldots$, with $T$ a symmetric operator in $\left[W_{n}\right]$.

In fact,

$$
\begin{aligned}
\mathscr{F}_{c}^{-1} \mathscr{F}_{c}\left(T(\cdot)^{n}\right)(z) & =\int_{B} \int_{B} T(x+i y+z)^{n} p_{c}(d x) p_{c}(d y) \\
& =T z^{n}+\int_{B} \int_{B} \sum_{j=0}^{n-1} T z^{j}(x+i y)^{n-j} p_{c}(d x) p_{c}(d y) \\
& =T z^{n} \quad \text { by Lemma 3.8. }
\end{aligned}
$$

(Step 2) By Proposition 3.4, we have

$$
f(z)=\sum_{n=0}^{\infty}(1 / n !) D^{n} f(0) z^{n} .
$$

Consequently,

$$
\mathscr{F}_{c} f(z)=\sum_{n=0}^{\infty}(1 / n !) \mathscr{F}_{c}\left(D^{n} f(0)(\cdot)^{n}\right)(z),
$$

by Lebesgue's dominated convergence theorem.

Finally, by Step 1 and the dominated convergence theorem again,

$$
\begin{aligned}
\mathscr{F}_{c}^{-1} \mathscr{F}_{c} f(z) & =\sum_{n=0}^{\infty}(1 / n !) \mathscr{F}_{c}^{-1} \mathscr{F}_{c}\left(D^{n} f(0)(\cdot)^{n}\right)(z) \\
& =\sum_{n=0}^{\infty}(1 / n !) D^{n} f(0) z^{n}=f(z) .
\end{aligned}
$$

REMARK 3. Combining 3.9 and 3.3, we see that $\mathscr{F}_{c}\left(\mathcal{E}_{a}\right)=\mathcal{E}_{a}$. 
THEOREM 3.10. $\mathcal{E}_{a}(B)$ is dense in $L^{2}\left(B, p_{c}\right)$.

REMARK 4. It is easy to see that if $f \in \mathcal{E}_{a}(B)$, then

$$
\begin{aligned}
& f_{y}: x \rightarrow f(x+y), \quad y \in B, \\
& f^{d}: x \rightarrow f(d x), \quad d \in R,
\end{aligned}
$$

are also in $\mathcal{E}_{a}(B)$.

Proof OF 3.10. By Remark 4, we may assume $c=1$.

Let $\left\{e_{j}: j=1,2, \ldots, n\right\}$ be a set $\subset B^{*}$. Define $P_{n}^{a}(x)=\Pi_{j=1}^{n}\left(x, e_{j}\right)^{a_{j}}$ where $a=\left(a_{1}, a_{2}, \ldots, a_{n}\right)$ and $a_{j}$ 's are positive integers. Obviously, $P_{n}^{a} \in \mathcal{E}_{a}(B)$ for all $a$ and $n$. Since the algebra generated by $P_{n}^{a}$ (i.e. the linear combination of products of $P_{n}^{a}$ 's) is dense in $L^{2}\left(B, p_{1}\right)$ (see, for example [7], [15]), our conclusion follows immediately.

Theorem 3.11 (PARSeVAl's formula). For all $f, g \in \mathcal{E}_{a}(B)$, we have

$$
\int_{B}\left(\mathscr{F}_{2} f\right)(y) g(y) p_{1}(d y)=\int_{B} f(y) \mathscr{F}_{2} g(y) p_{1}(d y) .
$$

Proof. It is not hard to see by direct computations and induction that (3) is true for $f(x)=A x^{m}$ and $g(x)=T x^{n}$ (where $A$ and $T$ are symmetric members in $W_{m}$ and $W_{n}$, respectively). The theorem then follows by Proposition 3.4 and Lebesgue's dominated convergence theorem. (This provides a more direct proof for the Parseval's formula without using the knowledge of the Hermite polynomials. See also Remark 6.)

Corollary 3.12. (i) $\int_{B} \mathscr{F}_{2} f(y) \cdot \mathscr{F}_{2} g(y) p_{1}(d y)=\int_{B} f(x) g(-x) p_{1}(d y)$,

(ii) $\int_{B} \mathscr{F}_{2} f(y) \cdot \overline{\mathscr{F}_{2} g(y)} p_{1}(d y)=\int_{B} f(x) \overline{g(x)} p_{1}(d x)$,

(iii) $\int_{B}\left|\mathscr{F}_{2} f(y)\right|^{2} p_{1}(d y)=\int_{B}|f(x)|^{2} p_{1}(d x)$.

Proof. (i) follows by Theorem 3.11. (ii) follows by the fact that $\overline{\mathscr{F}_{2} g(y)}=$ $\mathscr{F}_{2}^{-1} \bar{g}(y)$ and (iii) follows from (ii).

Notation. We customarily use $\mathscr{F}$ to stand for $\mathscr{F}_{2}$.

REMARK 5. Following [2], we may also extend the Fourier-Wiener transform to $L^{2}\left(B, p_{1}\right)$ in the following way.

If $f \in L^{2}\left(B, p_{1}\right)$ and $\left\{f_{n}\right\}$ is a sequence of functions in $\mathcal{E}_{a}(B)$ such that $f_{n} \rightarrow f$ in $L^{2}\left(B, p_{1}\right)$ (by 3.10 ), then we define the Fourier-Wiener transform of $f$ by $\mathscr{F} f=$ $L^{2}-\lim \mathscr{F} f_{n}$.

It follows by Corollary 3.12(iii) that $\mathscr{F}$ extends to a unitary operator on $L^{2}\left(B, p_{1}\right)$, i.e. $\|\mathscr{F} f\|_{L^{2}}=\|f\|_{L^{2}}$ for all $f \in L^{2}\left(B, p_{1}\right)$.

REMARK 6. Let $h_{n}=(-1)^{n}(n !)^{-1 / 2} e^{x^{2} / 2}\left(d^{n} / d x^{n}\right) e^{-x^{2} / 2}$ be the one-dimensional normalized Hermite polynomial of degree $n$. Let $e_{i}$ be an orthonormal basis of $H$ which lies in $B^{*}$. For any multiple indices $I=\left(i_{1}, i_{2}, \ldots, i_{n}\right)$, we define $h_{l}(x)=$ $\Pi_{k=1}^{n} h_{i_{k}}\left(\left(x, e_{k}\right)\right)$. Then $\left\{h_{I}\right\}$ forms an orthonormal basis in $L^{2}\left(B, p_{1}\right)$ (see [15]). Employing the computation of Hida [7], we get

$$
\mathscr{F} h_{I}(y)=i^{|I|} h_{I}(y), \quad y \in B \text {. }
$$


(This identity obviously also implies Theorem 3.11.) Furthermore, for any $f \in$ $L^{2}\left(B, p_{1}\right)$, we have $f=\Sigma_{I} a_{I} h_{I}\left(L^{2}\right)$ and by (4) we get

$$
\mathscr{F} f=\sum_{I} a_{I} i^{|I|} h_{I}\left(L^{2}\right)
$$

Since $h_{I} \in \mathcal{E}_{a}(B)$, if we take (5) as the definition of the Fourier-Wiener transform on $L^{2}\left(B, p_{1}\right),(5)$ is obviously equivalent to the definition given in Remark 5 .

4. Application I. Let $f$ be a function defined on $B$. We may regard $f$ as a function $g$ defined in a neighborhood of the origin of $H$ by restricting $f$ to the coset $x+H$ and defining $g(h)=f(x+h)$. If $g$ is $k$-times Fréchet-differentiable at 0 then we say that $f$ is $k$-times $H$-differentiable at $x$. We denote the $k$-times $H$-differentiation of $f$ at $x$ by $f^{(k)}(x)$. After Gross [6], if $f$ is a twice $H$-differentiable function on $B$ such that $f^{\prime \prime}(x)$ is a trace class operator on $H$ for each $x \in B$ then we define the Laplacian $\Delta f(x)$ by $\operatorname{trace}_{H} f^{\prime \prime}(x)$.

If $f$ is twice Fréchet-differentiable in $B$ then the second Fréchet-derivative $D^{2} f(x) \in L\left(B, B^{*}\right)$ and $f$ is automatically twice $H$-differentiable. Restricting to $H$, $\left.D^{2} f(x)\right|_{H}=f^{\prime \prime}(x)$, where $f^{\prime \prime}(x)$ is a symmetric member of $L(H, H)$ and therefore of trace class by Goodman's theorem (see [11]).

In this section, we apply the Fourier-Wiener transform to the Cauchy problem below.

$$
u_{t}(x, t)=-\Re^{k} u(x, t) ; \quad u(x, 0)=f(x) \quad(t>0, x \in B)
$$

where $k$ is any integer $\geqslant 1$, and

$$
\Re u(x)=-\Delta u(x)+(x, D u(x)) \quad \text { (if it exists). }
$$

If $u$ is twice Fréchet-differentiable in $B$, we may write

$$
\Delta u(x)=\operatorname{trace}_{H}\left[D^{2} u(x)\right]
$$

Our goal is to show that for $f \in \mathcal{E}_{a}(B)$, there exists a unique solution for (1) which is also in $\varepsilon_{a}(B)$.

Remark 1. When $k=1$, Piech [14] has shown the existence of a solution for equation (1) (with $f$ a Lip-1 function) such that $u(x, t) \rightarrow_{t \rightarrow 0} f(x)$ uniformly on bounded sets. In [15], she also studied the semigroup associated with $u_{t}=-\Re u$ on $L^{2}\left(B, p_{1}\right)$. A more general definition of $\Re$ which does not require the existence of both $\Delta u$ and $(x, D u(x))$ is also given in [15].

We shall separate our proof into two parts.

Part I (Existence). For $c>0$, we let

$$
\Re_{c} u(x)=-\Delta u(x)+c(x, D u(x))
$$

and

$$
\tilde{\mathscr{T}} u(x)=(x, D u(x))
$$

LEMMA 4.1. For $u \in \varepsilon_{a}$, we have

(i) $\Delta\left(\mathcal{E}_{a}\right) \subset \mathcal{E}_{a}, \tilde{\mathscr{T}}\left(\mathcal{E}_{a}\right) \subset \mathcal{E}_{a}$ and consequently, $\Re_{c}\left(\mathcal{E}_{a}\right) \subset \mathcal{E}_{a}$.

(ii) $\left(\mathscr{F}_{1 / c} \Re_{c} u\right)(y)=c \tilde{\mathscr{T}}\left(\mathscr{F}_{1 / c} u\right)(y)$ for $y \in B$ (or $\left.[B]\right)$. 
Proof. (i) Trivial.

(ii) Recall Remark 2 of $\S 3$. Let $f(x)=D u(x+i y)$ and $t=1 / c$ and apply Theorem 3.7. We get

$$
\int_{B}(x, D u(x+i y)) p_{1 / c}(d x)=c^{-1} \int_{B} \operatorname{trace}_{H}\left[D^{2} u(x+i y)\right] p_{1 / c}(d x) .
$$

On the other hand,

$$
\int_{B}(x, D u(x+i y)) p_{1 / c}(d x)
$$

(where the differentiation is with respect to $x$ )

$$
\begin{aligned}
& =\int_{B} \tilde{\mathscr{T}} u(x+i y) p_{1 / c}(d x)-i \int_{B}(y, D u(x+i y)) p_{1 / c}(d x) \\
& =\int_{B} \tilde{\mathscr{T}} u(x+i y) p_{1 / c}(d x)-\left(y, \int_{B} i D u(x+i y) p_{1 / c}(d x)\right)
\end{aligned}
$$

(by the definition of vector valued integral)

$$
\begin{aligned}
& =\int_{B} \tilde{\mathscr{T}} u(x+i y) p_{1 / c}(d x)-\left(y, \int_{B} D_{y} u(x+i y) p_{1 / c}(d x)\right) \\
& =\int_{B} \tilde{\mathscr{T}} u(x+i y) p_{1 / c}(d x)-\left(y, D\left[\int_{B} u(x+i y) p_{1 / c}(d x)\right]\right)
\end{aligned}
$$

(by the fact that $u \in \mathcal{E}_{a}$ and the dominated convergence theorem)

$$
=\mathscr{F}_{1 / c} \tilde{\mathcal{X}} u(y)-\tilde{\mathfrak{K}}\left(\mathscr{F}_{1 / c} u\right)(y) .
$$

Thus, $\left(\mathscr{F}_{1 / c} \Re_{c} u\right)(y)=c \tilde{\mathscr{T}}\left(\mathscr{F}_{1 / c} u\right)(y)$ (see also [10]).

Now, by taking the Fourier-Wiener transform and letting $v(y, t)=\left(\mathscr{F}_{1} u\right)(y, t)$, (1) becomes

$$
v_{t}(y, t)=-\tilde{\mathscr{T}}^{k} v(y, t) ; \quad v(y, 0)=\mathscr{F}_{1} f(y) \quad(y \in B, t>0)
$$

provided that

$$
v_{t}(y, t)=\left(\mathscr{F}_{1} u_{t}\right)(y, t)
$$

By Theorem 3.9, if we assume $f \in \mathcal{E}_{a}$, then it suffices to consider the Cauchy problem (6) and the condition (7).

LEMMA 4.2. Let $T \in W_{n}$ (T is not necessarily symmetric), and $f(x)=T x^{n}$. Then

$$
\tilde{\mathscr{T}} f(x)=n f(x), \quad x \in B(\text { or }[B]) \text {. }
$$

REMARK 2. The above lemma says that $\{1,2, \ldots\}$ are eigenvalues of $\tilde{\mathscr{N}}$ with the associated eigenvectors in $\mathcal{E}_{a}$. It is well known that $\{1,2, \ldots\}$ is the spectrum of $\Re$ when we regard $\Re$ as a (selfadjoint) operator on $L^{2}\left(B, p_{1}\right)$ (see, for example, [12], [14], [15]). Since $\mathcal{E}_{a}(B) \subset L^{2}\left(B, p_{1}\right)$, hence there is no eigenvector of $\tilde{\mathscr{T}}$ in $\varepsilon_{a}(B)$ with eigenvalue other than $\{1,2, \ldots\}$ (by Theorem 3.9).

Proof of 4.2. It is easy to see that

$$
D f(x) h=T x^{n-1} h+T x^{n-2} h x+\cdots+T h x^{n-1}, \quad x \in B .
$$


Consequently,

$$
\tilde{\mathscr{T}} f(x)=D f(x)(x)=n T x^{n}=n f(x) \text {. }
$$

LEMMA 4.3. Let $T$ be as in Lemma 4.2, and $u(x, t)=e^{-t n^{k}} T x^{n}$. Then

(a) $u(x, t)$ satisfies $u_{t}=-\tilde{\Upsilon}^{k} u$ and $u(x, 0)=T x^{n}$.

(b) $u(x, t) \rightarrow_{t \rightarrow 0} T x^{n}$ uniformly on bounded subsets of $[B]$.

Proof. By Lemma 4.2, $-\tilde{\Upsilon}^{k} u(x, t)=-n^{k} e^{-t n^{k}} T x^{n}=-n^{k} u(x, t)=u_{t}(x, t)$. This proves (a).

(b) is trivial.

Notation. We define for $f \in \mathcal{E}_{a}$

$$
\|f\|_{N}=\sup \{|f(x)|:\|x\|<N\}
$$

Obviously, $\|\cdot\|_{N}$ is a norm on $\varepsilon_{a}$.

THEOREM 4.4. Suppose $\left\{T_{n}\right\}$ is a sequence of bounded linear operators such that $T_{n} \in W_{n}, n=0,1,2, \ldots$ Assume that there exist constants $K$ and $M$ such that

$$
\left\|T_{n}\right\|_{w_{n}} \leqslant K M^{n}, \quad n=1,2, \ldots
$$

Then we have

(a) The series $\sum_{j=0}^{\infty}(1 / j !) T_{j} x^{j}$ converges absolutely and uniformly on every bounded subset of $[B]$ to a function $f$ in $\mathcal{E}_{a}$.

(b) If for $t \geqslant 0$, we let

$$
u(x, t)=\sum_{n=0}^{\infty}\left(e^{-t n^{k}} / n !\right) T_{n} x^{n}
$$

then this series converges absolutely, $x$-uniformly on bounded subsets and t-uniformly on $[0, \infty)$. Also $u(\cdot, t) \in \mathcal{E}_{a}$.

(c) For each $x \in[B], u(x, t) \in C^{\infty}([0, \infty))$.

(d) $u(x, t)$ satisfies $u_{t}(x, t)=-\tilde{\vartheta}^{k} u(x, t)$ and $u(x, 0)=f(x)$.

(e) $u(x, t) \rightarrow f(x)$ uniformly on bounded sets as $t \rightarrow 0$.

Proof. (a) Since

$$
\left\|T_{j} x^{j}\right\|_{N} \leqslant\left\|T_{j}\right\|_{w_{j}} N^{j} \leqslant K(M N)^{j}
$$

and

$$
\sum_{j=0}^{\infty}(1 / j !) K(M N)^{j}=K \exp (M N)<\infty
$$

hence, by the $M$-test $\sum_{j=0}^{\infty}(1 / j !) T_{j} x^{j}$ converges absolutely and uniformly on every bounded set. Consequently, for each pair of $x, y \in[B], \sum_{j=0}^{\infty}(1 / j !) T_{j}(x+\lambda y)^{j}$ converges $\lambda$-uniformly on compact subsets of C. Thus $f(x+\lambda y)$ is an entire function of $\lambda$. Moreover, it is easy to see that $|f(x)|<K \exp (M\|x\|)$ and (a) follows.

(b) Observe that $e^{-t n^{k}} \leqslant 1$, and so (b) follows immediately from (a).

(c) Since $\left|\sum_{n=0}^{\infty}\left(-n^{k}\right)^{m}\left(e^{-t n^{k}} / n !\right) T_{n} x^{n}\right|<\sum_{n=0}^{\infty} n^{k m}\left(K M^{n} / n !\right)\|x\|^{n}$ for every $m \geqslant 1$, hence $u(x, t) \in C^{\infty}([0, \infty))$ for each $x \in[B]$ and 


$$
\left(\partial^{m} / \partial t^{m}\right) u(x, t)=\sum_{n=0}^{\infty}\left(-n^{k}\right)^{m}\left(e^{-t n^{k}} / n !\right) T_{n} x^{n} .
$$

(d) Follows immediately by Lemma 4.3 , (c) and the fact that $\sum_{n=0}^{\infty}\left(n^{k} / n !\right)\left(K M^{n}\right)$ $<\infty$.

(e) Follows from Lemma 4.3 and (b).

Corollary 4.5. If $g \in \mathcal{E}_{a}$, we define

$$
u(x, t)=\sum_{n=0}^{\infty}\left(e^{-t n^{k}} / n !\right) D^{n} g(0) x^{n} \text { for } t \geqslant 0 .
$$

Then (i) $u(x, t)$ satisfies $u_{t}(x, t)=-\tilde{\mathscr{T}}^{k} u(x, t) ; u(x, 0)=g(x)$.

(ii) $u(x, t) \rightarrow_{t \rightarrow 0} g(x)$ uniformly on bounded sets in [B].

Next we establish a class of functions satisfying condition (7).

LEMMA 4.6. Suppose $u(x, t) \in \mathcal{E}_{a}$ for every $t \in\left[t_{1}, t_{2}\right] \subset \mathbf{R}$, and for every $x \in[B]$, $u(x, t)$ is a $C^{1}$-function of $t$ in $\left[t_{1}, t_{2}\right]$. Assume that for each $y \in[B]$, there is $a$ function $W^{*}(x) \in L^{1}\left(B, p_{c}\right)$ such that if $x \in B$

$$
|(\partial / \partial t) u(x+i y, t)| \leqslant W^{*}(x) \text { for all } t \text { in }\left[t_{1}, t_{2}\right] \text {. }
$$

Then we have

$$
(\partial / \partial t)\left(\mathscr{F}_{c} u\right)(y, t)=\mathscr{F}_{c}((\partial / \partial t) u)(y, t) .
$$

Proof. Suppose $t_{0}, t_{0}+h \in\left[t_{1}, t_{2}\right]$. Then

$$
\begin{aligned}
\mid u\left(x+i y, t_{0}+h\right) & -u\left(x+i y, t_{0}\right)|/| h \mid \\
& <\left|(\partial / \partial t) u\left(x+i y, t_{0}+\theta h\right)\right|, \quad \text { where } 0<\theta<1, \\
& \leqslant W^{*}(x) \in L^{1}\left(B, p_{c}\right), \quad \text { by }(12) .
\end{aligned}
$$

It follows by Lebesgue's dominated convergence theorem that

$$
\begin{aligned}
\lim _{h \rightarrow 0} h^{-1}\left(\mathscr{F}_{c} u\right. & \left.\left(y, t_{0}+h\right)-\mathscr{F}_{c} u\left(y, t_{0}\right)\right) \\
& =\lim _{h \rightarrow 0} \int_{B} h^{-1}\left(u\left(x+i y, t_{0}+h\right)-u\left(x+i y, t_{0}\right)\right) p_{c}(d x) \\
& =\int_{B} \lim _{h \rightarrow 0} h^{-1}\left(u\left(x+i y, t_{0}+h\right)-u\left(x+i y, t_{0}\right)\right) p_{c}(d x) \\
& =\int_{B}(\partial / \partial t) u\left(x+i y, t_{0}\right) p_{c}(d x) \\
& =\mathscr{F}_{c}((\partial / \partial t) u)\left(y, t_{0}\right) .
\end{aligned}
$$

TheOREM 4.7. Suppose $f \in \mathcal{E}_{a}$ and $g(y)=\mathscr{F}_{1} f(y)$. Define

$$
u(y, t)=\sum_{n=0}^{\infty}\left(e^{-t n^{k}} / n !\right) D^{n} g(0) y^{n}
$$

and

$$
v(x, t)=\mathscr{F}_{1}^{-1} u(x, t)
$$


Then (i) $v(x, t)$ solves the Cauchy problem (1) i.e. $v_{t}(x, t)=-\mathscr{T}^{k} v(x, t) ; v(x, 0)=$ $f(x)$.

(ii) $v(x, t) \rightarrow_{t \rightarrow 0} f(x)$ uniformly on bounded sets.

Proof. Obviously, $v(x, 0)=\mathscr{F}_{1}^{-1} u(x, 0)=f(x)$. By Corollary 4.5 and Proposition 3.3, we have $g \in \mathcal{E}_{a}$ and $u_{t}(y, t)=-\tilde{\mathscr{T}}^{k} u(y, t)$. Employing Lemma 4.1, we get $\left(\mathscr{F}_{1}^{-1} \tilde{\mathscr{T}}^{k} u\right)(x, t)=\mathscr{T}^{k} v(x, t)$. Now, to prove (i), it remains to show that

$$
\left(\mathscr{F}_{1}^{-1} u_{t}\right)(x, t)=v_{t}(x, t) \text {. }
$$

In fact, by 4.4(b), (c), we have

$$
\begin{aligned}
u_{t}(y, t) & =\sum_{n=0}^{\infty} e^{-t n^{k}}\left(-n^{k} / n !\right) D^{n} g(0) y^{n} \\
& =\frac{1}{2 \pi i} \int_{|\lambda|=2}\left(\sum_{n=0}^{\infty} e^{-n^{k}}\left(-n^{k} / \lambda^{n+1}\right)\right) g(\lambda y) d \lambda .
\end{aligned}
$$

It follows that $\left|u_{t}(y, t)\right| \leqslant\left(\sum_{n=0}^{\infty} n^{k} / 2^{n}\right) K_{1} \exp \left(2 K_{2}\|y\|\right)$, where $K_{1}, K_{2}>0$ are those constants such that $|g(z)| \leqslant K_{1} \exp \left(K_{2}\|z\|\right)$. In particular, for each $y \in[B]$, say $\|y\| \leqslant c_{1}$, we have for $x \in B$

$$
\left|u_{t}(x+i y, t)\right| \leqslant\left(\sum_{n=0}^{\infty} n^{k} / 2^{n}\right) K_{1} \exp \left(2 K_{2} c_{1}\right) \exp \left(2 K_{2}\|x\|\right) \in L^{1}\left(B, p_{1}\right) .
$$

Therefore condition (12) is fulfilled and (*) follows by Lemma 4.6. This proves (i).

(ii) Let $\|x\| \leqslant c_{1}$; we have

$$
\begin{aligned}
|v(x, t)-f(x)| & =\left|\mathscr{F}_{1}^{-1} u(x, t)-\mathscr{F}_{1}^{-1} g(x)\right| \\
& \leqslant \frac{1}{2 \pi}\left|\int_{B} \int_{|\lambda|=2}\left(\sum_{n=0}^{\infty}\left(e^{-n^{k_{t}}}-1\right) / \lambda^{n+1}\right) g(\lambda(y-i x)) d \lambda p_{1}(d y)\right| \\
& \leqslant K_{1} t\left(\sum_{n=0}^{\infty} n^{k} / 2^{n}\right) \exp \left(2 K_{2} c_{1}\right)\left(\int_{B} \exp \left(2 K_{2}\|y\|\right) p_{1}(d y)\right) \\
& \rightarrow 0 \text { uniformly on }\left\{\|x\| \leqslant c_{1}\right\} .
\end{aligned}
$$

REMARK 3. We note that the formula (11) in Corollary 4.5 can also be written as

$$
u(x, t)=(1 / 2 \pi i) \int_{|\lambda|=2}\left(\sum_{n=0}^{\infty} e^{-n^{k_{t}} / \lambda^{n+1}}\right) g(\lambda x) d \lambda
$$

and (13) also has the following form

$$
\begin{aligned}
v(x, t) & =\mathscr{F}_{1}^{-1} u(x, t) \\
& =\frac{1}{2 \pi i} \int_{B} \int_{B}\left[\int_{|\lambda|=2}\left(\sum_{n=0}^{\infty} e^{-n^{k_{t}}} / \lambda^{n+1}\right) f(z+\lambda x+i \lambda y) d \lambda\right] p_{1}(d z) p_{1}(d y) .
\end{aligned}
$$

When $k=1$, the solution of (1) is also given by

$$
\begin{aligned}
v(x, t) & =\mathscr{F}_{1}^{-1}\left(g\left(e^{-t} y\right)\right)(x) \\
& =\int_{B} \int_{B} f\left(z+e^{-t} x+i e^{-t} y\right) p_{1}(d y) p_{1}(d z) .
\end{aligned}
$$


It can be shown that the integral (15) can also be written as

$$
\int_{B} f\left(e^{-t} x+\sqrt{1-e^{-2 t}} y\right) p_{1}(d y)=\int_{B} f(y) o_{t}(x, d y),
$$

where $o_{t}(x, E)=p_{1-e^{-2 t}}\left(e^{-t} x, E\right)$.

We note that this is a well-known formula (see [14], [15]), and that the measures $o_{t}(x, d y)$ are the transition measures of the infinite dimensional Ornstein-Uhlenbeck process.

Part II (Semigroup theory and uniqueness). First of all, we shall study the semigroup induced by the solution of the Cauchy problem (6). Using this as a tool, we are able to show that there is only one solution in $\tilde{\mathcal{E}}_{a}$ (see Definition 4.8 ) for (6) under some $t$-smoothness assumption (see 4.14). Then the uniqueness of (1) follows by applying the Fourier-Wiener transform.

Definition 4.8. Denote by $\tilde{\varepsilon}_{a}$ the space of analytic functions $f$ defined on $[B]$ such that $\|f\|_{N}=\sup _{\widetilde{\sigma}}\{|f(z)|:\|z\| \leqslant N\}$ is finite for every $N=1,2,3, \ldots$

Obviously, $\tilde{E}_{a} \subset \tilde{\mathscr{E}}_{a}$.

Proposition 4.9. (i) $\|f\|_{N}$ is a norm on $\tilde{E}_{a}$ and $\|f\|_{N} \leqslant\|f\|_{N+1}$ for $N=$ $1,2,3, \ldots$.

(ii) With the topology induced by $\|\cdot\|_{N}, \tilde{\varepsilon}_{a}$ forms a (sequentially) complete metrizable locally convex space.

Proof. (i) Trivial.

(ii) Since $\|\cdot\|_{N}$ is a sequence of norms which separate "points" of $\tilde{\mathscr{E}}_{a}$, hence $\tilde{\mathscr{E}}_{a}$ forms a metrizable locally convex space. It remains to show that $\tilde{E}_{a}$ is complete. Suppose $\left\{f_{n}\right\}$ is a Cauchy sequence in $\tilde{\mathscr{E}}_{a}$. Then for each $N,\left\{f_{n}\right\}$ is also a Cauchy sequence on $\{\|z\| \leqslant N\}$; hence $\left\{f_{n}\right\}$ converges uniformly on $\{\|z\|<N\}$ to a function, say $f^{N}$. We thereby define a function $f$ such that $f=f^{N}$ on $\{\|z\|<N\}$. Since $\|\cdot\|_{N} \leqslant\|\cdot\|_{N+1}, f$ is well defined and $f_{n} \rightarrow f$ uniformly on every bounded set. It follows that $f$ is analytic. Moreover, for each fixed $N,\left\|f_{n}\right\|_{N} \leqslant$ some constant $C_{1}$ for all $n$. We conclude that $\|f\|_{N}<C_{1}$, i.e. $f \in \tilde{\varepsilon}_{a}$.

LEMMA 4.10. Every $f \in \tilde{\mathcal{E}}_{a}$ has a Maclaurin expansion which converges absolutely and uniformly on bounded sets.

Proof. Since $f(\lambda x)$ has a Maclaurin expansion in $\lambda$ for each $x \in[B]$ we have $f(x)=\sum_{n=0}^{\infty}(1 / n !) D^{n} f(0) x^{n}$. Furthermore,

$$
(1 / n !) D^{n} f(0) x^{n}=(1 / 2 \pi i) \int_{|z|=2} f(z x) / z^{n+1} d z
$$

Consequently, for $\|x\| \leqslant N$ we have

and

$$
(1 / n !)\left|D^{n} f(0) x^{n}\right| \leqslant 2^{-n}\|f\|_{2 N}
$$

$$
\sum_{n=0}^{\infty}(1 / n !)\left|D^{n} f(0) x^{n}\right| \leqslant\left(\sum_{n=0}^{\infty} 2^{-n}\right)\|f\|_{2 N}=2\|f\|_{2 N} .
$$

Thus we complete the proof. 
Proposition 4.11. $\tilde{\mathcal{E}}_{a}$ is the completion of $\mathcal{E}_{a}$.

Proof. It suffices to show that $\mathcal{E}_{a}$ is dense in $\tilde{\mathscr{E}}_{a}$. In fact, given any function $f \in \tilde{\mathcal{E}}_{a}$, Lemma 4.10 implies that as $p \rightarrow \infty, \Sigma_{n=0}^{p}(1 / n !) D^{n} f(0) x^{n}\left(\in \mathcal{E}_{a}\right)$ converges to $f$ uniformly on every bounded set. Thus $\varepsilon_{a}$ is dense in $\tilde{\varepsilon}_{a}$.

Now we define a family of operators $\left\{T_{t}\right\}$ on $\tilde{\varepsilon}_{a}$ in the following way.

Let $k$ be the integer in the Cauchy problem (1) and let $f \in \tilde{E}_{a}$. For $t>0$, we define

$$
T_{t} f(x)=\sum_{n=0}^{\infty}\left(e^{-n^{k_{t}}} / n !\right) D^{n} f(0) x^{n}
$$

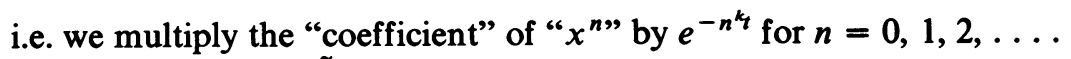

Obviously, $T_{t} f \in \tilde{\mathcal{E}}_{a}$ and by (16) we also have

$$
\left\|T_{\imath} f\right\|_{N} \leqslant \sup _{\|x\|<N}\left\{\sum_{n=0}^{\infty}(1 / n !) D^{n} f(0) x^{n}\right\}<2\|f\|_{2 N} .
$$

Hence $T_{t}$ 's are continuous, i.e. $T_{t} \in L\left(\tilde{\mathscr{E}}_{a}, \tilde{\mathscr{E}}_{a}\right)$.

Proposition 4.12. $\left\{T_{t}\right\}$ is an equicontinuous semigroup of class $\left(C_{0}\right)$ [20, Chapter 9].

Proof. Obviously,

$$
T_{t+s}=T_{t} T_{s} \text { and } T_{0}=I .
$$

By the mean value theorem, we have (for $t_{0}>0$ )

$$
\begin{aligned}
\left\|T_{t} f-T_{t_{0}} f\right\|_{N} & <\sum_{n=0}^{\infty}(1 / n !)\left|e^{-n^{k_{t}}}-e^{-n^{k_{t_{0}}}}\right|\left\|D^{n} f(0) x^{n}\right\|_{N} \\
& \leqslant\left|t-t_{0}\right|\|f\|_{2 N}\left(\sum_{n=1}^{\infty} n^{k} / 2^{n}\right)
\end{aligned}
$$

by (16). Thus

$$
\lim _{t \rightarrow t_{0}} T_{t} f=T_{t_{0}} f \text { for } t_{0}>0 .
$$

Finally, suppose $q$ is any continuous seminorm on $\tilde{\varepsilon}_{a}$. Then there exist $N$ and a constant $C_{1}$ such that $q(f) \leqslant C_{1}\|f\|_{N}$. Consequently, $q\left(T_{\imath} f\right)<C_{1}\left\|T_{\imath} f\right\|_{N}<$ $2 C_{1}\|f\|_{2 N}$, i.e. $\left\{T_{t}\right\}$ is equicontinuous.

Proposition 4.13. $-\tilde{\mathscr{T}}^{k}$ is the infinitesimal generator of $\left\{T_{t}\right\}$ and the domain of $-\tilde{\mathscr{T}}^{k}=\tilde{\tilde{E}}_{a}$.

Proof. Let $f \in \tilde{\mathscr{E}}_{a}$ and $|h|<1$. Then for each integer $N$ we have

$$
\begin{aligned}
\left\|h^{-1}\left(T_{h}-I\right) f+\tilde{\Upsilon}{ }^{k} f\right\|_{N} & \leqslant \sup _{\|x\|<N}\left[\sum_{n=1}^{\infty}\left|\left(e^{-n^{k} h}-1\right) / h+n^{k}\right|\left|D^{n} f(0) x^{n} / n !\right|\right] \\
& \leqslant(|h| / 2)\left(\sum_{n=1}^{\infty} n^{2 k} / 2^{n}\right)\|f\|_{2 N} \rightarrow 0 \text { as } h \rightarrow 0 .
\end{aligned}
$$

Therefore, $\lim _{h \rightarrow 0} h^{-1}\left(T_{h}-I\right) f$ exists and equals $-\tilde{\mathscr{T}}^{k} f$. 
REMARK 4. As a consequence (see Yosida [20]), $\tilde{\mathscr{T}}^{k}$ is a closed operator on $\tilde{\mathscr{E}}_{a}$. Furthermore, $T_{t}$ can be uniquely represented by

$$
T_{\imath} f=\lim _{n \rightarrow \infty} \exp \left(t \tilde{\mathscr{T}}^{k}\left(I-n^{-1} \tilde{\mathscr{T}}^{k}\right)^{-1}\right) f
$$

THEOREM 4.14. There is a unique solution $v(y, t)$ for the Cauchy problem (6) such that (i) $v(\cdot, t) \in \tilde{\mathcal{E}}_{a}$ for each $t \geqslant 0$, (ii) $v(y, t)$ is (strongly) differentiable in $t$ (i.e. $(\partial / \partial t) v(y, t)$ exists uniformly on bounded sets). Moreover, the solution is given by $v(y, t)=T_{t}\left(\mathscr{F}_{1} f\right)(y)$.

Proof. Let $g(y, s)$ be any solution for (6) such that (i) and (ii) hold.

Fix $t>0$. Set $F(y, s)=T_{t-s} g(y, s)$ for $t>s>0, y \in[B]$. Since $g(\cdot, s) \in \tilde{E}_{a}$, the domain of $-\tilde{\mathscr{T}}^{k}$, it follows by [20, Chapter 9, Theorem 2] that $F(y, s)$ is strongly differentiable and

$$
(\partial / \partial s) F(y, s)=\tilde{\mathscr{\imath}}^{k} T_{t-s} g(y, s)+T_{t-s}(\partial / \partial s) g(y, s)
$$

(since $T_{t}$ is an equicontinuous semigroup and $g$ satisfies (ii))

$$
\begin{aligned}
& =T_{t-s} \tilde{\mathscr{T}}^{k} g(y, s)-T_{t-s} \tilde{\mathfrak{T}}^{k} g(y, s) \\
& =0, \quad y \text {-uniformly on bounded sets. }
\end{aligned}
$$

This implies that $F(y, s)=C$ (a constant) on $[B]$ for $0<s<t$. In particular, $F(y, t)=F(y, 0)=T_{t} g(y, 0)=T_{t}\left(\mathscr{F}_{1} f\right)(y)$. On the other hand, $F(y, t)=$ $T_{0} g(y, t)=g(y, t)$. We conclude that $g(y, t)=T_{t}\left(\mathscr{F}_{1} f\right)(y)$.

COROLlary 4.15. The Cauchy problem (1) has a unique solution $u(x, t)$ such that (i) $u(\cdot, t) \in \mathcal{E}_{a}$ for each $t \geqslant 0$, (ii) $u(x, t)$ is a (strongly) $C^{1}$-function of $t$ with the property that for $b, N>0, \sup _{\|y\|<N} \sup _{0<t<b}\left|u_{t}(x+i y, t)\right|$, as a function of $x$, belongs to $L^{1}\left(B, p_{1}\right)$.

Proof. Recalling Theorem 3.9, it suffices to show that $\mathscr{F}_{1} u(y, t)$ satisfies the conditions (i) and (ii) of Theorem 4.14. Obviously, by Proposition 3.3 and (i), $\mathscr{F}_{1} u(y, t)$ satisfies condition (i) of Theorem 4.14. To show that $\mathscr{F}_{1} u(y, t)$ satisfies condition (ii) of Theorem 4.14, we let $v(y, t)=\mathscr{F}_{1} u(y, t)$ and write

$$
\varepsilon^{-1}(v(y, t+\varepsilon)-v(y, t))=\int_{B} \varepsilon^{-1}(u(x+i y, t+\varepsilon)-u(x+i y, t)) p_{1}(d x)
$$

Since

$$
\left|\varepsilon^{-1}(u(x+i y, t+\varepsilon)-u(x+i y, t))\right|=\left|u_{t}(x+i y, t+\theta(x, y, t) \varepsilon)\right|
$$

where $0<\theta(x, y, t)<1$, it follows that if $\varepsilon<1$

$$
\sup _{\|y\|<N} \varepsilon^{-1}|u(x+i y, t+\varepsilon)-u(x+i y, t)|<\sup _{\|y\|<N} \sup _{0<s<1+t}\left|u_{s}(x+i y, s)\right| .
$$


Hence, by the property (ii) and Lebesgue's dominated convergence theorem, we get

$$
\begin{aligned}
\lim _{\varepsilon \rightarrow 0}\left\|\varepsilon^{-1}(v(y, t+\varepsilon)-v(y, t))-v_{t}(y, t)\right\|_{N} \\
\quad \leqslant \lim _{\varepsilon \rightarrow 0} \int_{B} \sup _{\|y\|<N}\left|\varepsilon^{-1}(u(x+i y, t+\varepsilon)-u(x+i y, t))-u_{t}(x+i y, t)\right| p_{1}(d x) \\
\quad=\int_{B} \lim _{\varepsilon \rightarrow 0} \sup _{\|y\|<N}\left|\varepsilon^{-1}(u(x+i y, t+\varepsilon)-u(x+i y, t))-u_{t}(x+i y, t)\right| p_{1}(d x) \\
\quad=0 .
\end{aligned}
$$

Moreover,

$$
\begin{aligned}
\lim _{\varepsilon \rightarrow 0} \| \int_{B}\left(u_{t}(x\right. & \left.+i y, t+\varepsilon)-u_{t}(x+i y, t)\right) p_{1}(d x) \|_{N} \\
& \leqslant \lim _{\varepsilon \rightarrow 0} \int_{B} \sup _{\|y\|<N}\left|u_{t}(x+i y, t+\varepsilon)-u_{t}(x+i y, t)\right| p_{1}(d x) \\
& =\int_{B} \lim _{\varepsilon \rightarrow 0} \sup _{\|y\|<N}\left|u_{t}(x+i y, t+\varepsilon)-u_{t}(x+i y, t)\right| p_{1}(d x) \\
& =0,
\end{aligned}
$$

by the condition (ii) and the dominated convergence theorem.

Thus $v(y, t)=\mathscr{F}_{1} u(y, t)$ satisfies (ii) of Theorem 4.14.

As a further application of the Fourier-Wiener transform, Corollaries 4.5 and 4.15, we may consider the following nonhomogeneous Cauchy problem.

$$
w_{t}(x, t)+\Re^{k} w(x, t)=h(x, t) ; \quad w(x, 0)=f(x),
$$

where $f(x) \in \varepsilon_{a}$ and $h(x, t)$ is a sufficiently smooth function of $t$ such that $h(\cdot, t) \in \mathcal{E}_{a}$. More precisely, we have

THEOREM 4.16. Suppose $h(x, t):[B] \times[0, \infty) \rightarrow C$ has the following three properties: (i) $h(x+\lambda y, t)$ is an entire function of $\lambda$ for each $t \in[0, \infty]$ and $x, y \in[B]$. (ii) Let $b>0$. There exist constants $K_{1}, K_{2}$ such that $\sup _{0<t<b}|h(x, t)| \leqslant K_{1} \exp \left(K_{2}\|x\|\right)$ for every $x \in[B]$. (iii) $h(x, t)$ satisfies property (ii) of Corollary 4.15.

Then there exists a unique solution $w(x, t)$ for the Cauchy problem (20) such that $w(x, t)$ satisfies (i), (ii) of Corollary 4.15. Moreover, $w(x, t)$ is given by

$$
w(x, t)=\mathscr{F}_{1}^{-1}\left(T_{t}\left(\mathscr{F}_{1} f\right)\right)(x)+\int_{0}^{t} \mathscr{F}_{1}^{-1}\left(T_{t-s}\left(\mathscr{F}_{1} h\right)\right)(x) d s
$$

Proof. By similar arguments as in the proof of Proposition 3.3 we have

(a) $\mathscr{F}_{1} h(y, t), \mathscr{F}_{1}^{-1} h(y, t)$ also have the properties (i) and (ii).

(b) For every $t \geqslant 0, h(x, t) \in \mathcal{E}_{a}$ (by (i), (ii)).

(c) $\sup _{0<t<b}\left\|D^{n} h(x, t)\right\|_{\left[W_{n}\right]} \leqslant K_{1} \exp \left(K_{2} n\right) \exp \left(K_{2}\|x\|\right)$.

Next, by the same argument as in the proof of Corollary 4.15, we have

(d) $\mathscr{F}_{1} h(y, t)$ is a (strongly) $C^{1}$-function of $t$ (by all three properties (i)-(iii)).

To finish the proof, we consider the following Cauchy problem (which is the Fourier-Wiener 1-transform of (20)):

$$
\tilde{w}_{t}(y, t)+\tilde{\mathscr{T}}^{k} \tilde{w}(y, t)=\tilde{h}(y, t) ; \quad \tilde{w}(y, 0)=\tilde{f}(y),
$$

where "“" means the Fourier-Wiener 1-transform. 
We claim that $\tilde{w}(y, t)=T_{t} \tilde{f}(y)+\int_{0}^{t} T_{t-s} \tilde{h}(y, s) d s$ is the unique solution of (22) satisfying conditions (i), (ii) of Theorem 4.14. In fact, if we let $G(y, t)=$ $\int_{0}^{t} T_{t-s} \tilde{h}(y, s) d s$, then

$$
\begin{aligned}
\frac{1}{\varepsilon}(G(y, t+\varepsilon)-G(y, t))= & \frac{1}{\varepsilon}\left(\int_{0}^{t+\varepsilon} T_{t+\varepsilon-s} \tilde{h}(y, s) d s-\int_{0}^{t} T_{t-s} \tilde{h}(y, s) d s\right) \\
= & \varepsilon^{-1} \int_{t}^{t+\varepsilon} T_{t+\varepsilon-s} \tilde{h}(y, s) d s \\
& +\varepsilon^{-1}\left(T_{e}-I\right)\left(\int_{0}^{t} T_{t-s} \tilde{h}(y, s) d s\right) .
\end{aligned}
$$

Since $\tilde{h}(y, s)$ is (strongly) continuous,

$$
\lim _{\varepsilon \rightarrow 0} \varepsilon^{-1} \int_{t}^{t+\varepsilon} T_{t+\varepsilon-s} \tilde{h}(y, s) d s=T_{0} \tilde{h}(y, t)=\tilde{h}(y, t)
$$

( $y$-uniformly on bounded sets). Since $\int_{0}^{t} T_{t-s} \tilde{h}(y, s) d s \in \tilde{\mathscr{E}}_{a}$, we have

$$
\lim _{\varepsilon \rightarrow 0} \varepsilon^{-1}\left(T_{e}-I\right)\left(\int_{0}^{t} T_{t-s} \tilde{h}(y, s) d s\right)=-\tilde{\mathscr{T}}^{k}\left(\int_{0}^{t} T_{t-s} \tilde{h}(y, s) d s\right) .
$$

Thus $G(y, t)$ is (strongly) differentiable and

$$
(\partial / \partial t) G(y, t)=-\tilde{\mathscr{T}}^{k}\left(\int_{0}^{t} T_{t-s} \tilde{h}(y, s) d s\right)+\tilde{h}(y, t) .
$$

As a result,

$$
(\partial / \partial t) \tilde{w}(y, t)=-\tilde{\mathscr{T}}^{k}\left(T_{t} \tilde{f}(y)\right)-\tilde{\mathscr{T}}^{k}\left(\int_{0}^{t} T_{t-s} \tilde{h}(y, s) d s\right)+\tilde{h}(y, t),
$$

or $\tilde{w}_{t}(y, t)+\tilde{\mathfrak{T}}^{k} \tilde{w}(y, t)=\tilde{h}(y, t)$. Obviously, $\tilde{w}(y, 0)=\tilde{f}(y)$.

By (23) and Theorem 4.14, the uniqueness follows. Finally, by taking the inverse Fourier-Wiener transform, the solution of the Cauchy problem is represented by

$$
w(x, t)=\mathscr{F}_{1}^{-1} T_{t} \tilde{f}(x)+\mathscr{F}_{1}^{-1}\left(\int_{0}^{t} T_{t-s} \tilde{h}(y, s) d s\right)(x, t) .
$$

Note that

$$
\begin{aligned}
\left|T_{t-s} \tilde{h}(y-i x, s)\right| & \leqslant \sum_{n=0}^{\infty}\left|(1 / n !) D^{n} \tilde{h}(0, s)(y-i x)^{n}\right| \\
& \leqslant K_{1} \sum_{n=0}^{\infty}(1 / n !) \exp \left(K_{2} n\right)(\|y\|+\|x\|)^{n} \\
& \quad \text { for } 0 \leqslant s \leqslant t,(\text { by (c) }) \\
& =K_{1} \exp \left(e^{K_{2}}\|x\|\right) \exp \left(e^{K_{2}}\|y\|\right) \in L^{1}\left(B, p_{1}(d y)\right) .
\end{aligned}
$$

It follows by Fubini's theorem that

$$
\mathscr{F}_{1}^{-1}\left(\int_{0}^{t} T_{t-s} \tilde{h}(y, s) d s\right)(x, t)=\int_{0}^{t} \mathscr{F}_{1}^{-1}\left(T_{t-s} \tilde{h}\right)(x, s) d s .
$$

Hence, $w(x, t)=\mathscr{F}_{1}^{-1}\left(T_{t} \mathscr{F}_{1} f\right)(x)+\int_{0}^{t} \mathscr{F}_{1}^{-1}\left(T_{t-s} \mathscr{F}_{1} h\right)(x, s) d s$. Of course, $w(x, 0)=$ $\mathscr{F}_{1}^{-1}\left(T_{0} \mathscr{F}_{1} f\right)(x)=f(x)$. Thus we complete the proof of Theorem 4.16.

REMARK 5. We note that all functions of the form $h(x, t)=P(t) f(x)$ where $P(t)$ is $C^{1}$ and $f \in \mathcal{E}_{a}$, satisfying the hypotheses of Theorem 4.16. Recall that $\mathcal{E}_{a}(B)$ is 
dense in $L^{2}\left(B, p_{1}\right)$, so we have a rich class of functions $h(x, t)$ satisfying (i)-(iii) of Theorem 4.16.

REMARK 6. Since $\mathscr{F}_{1}^{-1}\left(T_{t-s} \mathscr{F}_{1} h\right)(x, s)$ also has the property (ii), we see that

$$
\lim _{t \rightarrow 0} \int_{0}^{t} \mathscr{F}_{1}^{-1}\left(T_{t-s} \mathscr{F}_{1} h\right)(x, s) d s=0
$$

uniformly on bounded sets. Therefore, $w(x, t) \rightarrow f(x)$ also uniformly on bounded sets.

5. Application II. We shall consider in the following two other types of equations associated with the operator $\Re$. The first one is

$$
-\vartheta^{k} u(x)=f(x)
$$

and the second one is the Cauchy problem

$$
u_{t t}(x, t)=-\Re^{k} u(x, t) ; \quad u(x, 0)=f_{1}(x), \quad u_{t}(x, 0)=f_{2}(x) .
$$

By means of the Fourier-Wiener transform, our approach is very much the same as that of §4. Uniqueness of solutions of (1) and (2) and the nonhomogeneous problem associated with (2) will also be considered.

THEOREM 5.1. (i) In order that equation (1) has a solution in $\mathcal{E}_{a}$, it is necessary and sufficient that $f \in \mathcal{E}_{a}$ and $\mathcal{F}_{1} f(0)=0$. (ii) The solution of (1) is unique in the sense that if $W_{1}(x), W_{2}(x) \in \mathcal{E}_{a}$ and each solves (1) then $W_{1}-W_{2}$ is a constant.

Proof. (i) a. Necessity. Since $\Re\left(\mathcal{E}_{a}\right) \subset \mathcal{E}_{a}$ (Lemma 4.1), if $u(x)$ is a solution in $\mathcal{E}_{a}$ then $\mathcal{T}^{k} u(x) \in \mathcal{E}_{a}$ so does $f$.

Next, applying the Fourier-Wiener transform to (1), we get

$$
\text { - } \tilde{\mathscr{T}}^{k}\left(\mathscr{F}_{1} u\right)(y)=\mathscr{F}_{1} f(y) .
$$

Therefore $\mathscr{F}_{1} f(0)=-\tilde{\mathscr{T}}^{k}\left(\mathscr{F}_{1} u\right)(0)=0$.

b. Sufficiency (existence). Suppose $f \in \mathcal{E}_{a}$ and $\mathscr{F}_{1} f(0)=0$. We let $g(y)=\mathscr{F}_{1} f(y)$. Since $g \in \mathcal{E}_{a}, g(y)=\sum_{n=1}^{\infty}(1 / n !) D^{n} g(0) y^{n}($ since $g(0)=0)$. It is easy to see that

$$
v(y)=\mathscr{F}_{1} u(y)=v(0)-\sum_{n=1}^{\infty}\left(1 / n^{k} n !\right) D^{n} g(0) y^{n}
$$

solves equation (3). Consequently,

$$
u(x)=\mathscr{F}_{1}^{-1} v(x)=v(0)-\mathscr{F}_{1}^{-1}\left(\sum_{n=1}^{\infty}\left(1 / n^{k} n !\right) D^{n} g(0) y^{n}\right)(x)
$$

solves (1).

(ii) Uniqueness. Observe that if $h \in \mathcal{E}_{a}$, then $\tilde{\mathscr{T}} h=0$ iff $h \equiv \mathrm{a}$ const. It follows that if $W_{1}, W_{2}$ are two solutions for (1) such that $W_{1}, W_{2} \in \mathcal{E}_{a}$ and we let $H=W_{1}-W_{2}$, then we must have $\Re H=0$; hence $\tilde{\mathscr{T}}\left(\mathscr{F}_{1} H\right) \equiv 0$.

According to our observation $\mathscr{F}_{1} H \equiv C_{1}$, a constant. Therefore $H \equiv \mathscr{F}_{1}^{-1}\left(C_{1}\right) \equiv$ $C_{1}$.

REMARK 1. Note that if $f \in \mathcal{E}_{a}$ with $\mathscr{F}_{1} f(0)=0$, then for $x \in[B]$ we have $\left|T_{t}\left(\mathscr{F}_{1} f\right)(y-i x)\right| \leqslant\left(K_{1} e^{K_{2}\|x\|}\right) e^{-t} e^{K_{2}\|y\|}$ for some constants $K_{1}, K_{2}$ and $y \in B$. Observing that $\int_{B} \int_{0}^{\infty} e^{-t} \exp \left(K_{2}\|y\|\right) p_{1}(d y) d t<\infty$, we may also write (4) (by Fubini's theorem) as 


$$
u(x)=\int_{B} u(y) p_{1}(d y)+\int_{0}^{\infty} \mathscr{F}_{1}^{-1}\left(T_{t}\left(\mathscr{F}_{1} f\right)\right)(x) d t,
$$

where $T_{t}$ is defined by (17) of $\S 4$.

In the remainder of this section, we first show the existence of a solution for (2). Then by means of semigroup theory, we are able to prove uniqueness and also obtain the solution of the nonhomogeneous equation

$$
u_{t t}(x, t)+\mathcal{T}^{k} u(x, t)=h(x, t) ; \quad u(x, 0)=f_{1}(x), \quad u_{t}(x, 0)=f_{2}(x) .
$$

The following theorem is straightforward; we omit the proof.

TheOREM 5.2. Assume $g_{1}, g_{2}$ are functions in $\tilde{\mathcal{E}}_{a}$. Define

$$
v(y, t)=\sum_{n=0}^{\infty}\left(P_{n}(t) / n !\right) D^{n} g_{1}(0) y^{n}+\sum_{n=0}^{\infty}\left(Q_{n}(t) / n !\right) D^{n} g_{2}(0) y^{n},
$$

where $P_{n}(t)=\cos (\sqrt{n})^{k} t, \quad Q_{0}(t)=t, \quad Q_{n}(t)=(\sqrt{n})^{-k} \sin (\sqrt{n})^{k} t$ for $n=$ $1,2,3, \ldots$ Then for $t \in R$,

(i) $v_{t t}(y, t)=-\tilde{T}^{k} v(y, t) ; v(y, 0)=g_{1}(y)$ and $v_{t}(y, 0)=g_{2}(y)$.

(ii) As $t \rightarrow 0, v(y, t) \rightarrow g_{1}(y), v_{t}(y, t) \rightarrow g_{2}(y)$ uniformly on bounded sets in $[B]$.

TheOrem 5.3. Assuming $f_{1}, f_{2} \in \mathcal{E}_{a}$ and $g_{j}=\mathscr{F}_{1} f_{j}(j=1,2)$, we define $u(x, t)=$ $\mathscr{F}_{1}^{-1} v(x, t)$, where $v$ is given by (6). Then

(i) $u(x, t)$ solves the Cauchy problem (2).

(ii) As $t \rightarrow 0, u(x, t) \rightarrow f_{1}(x), u_{t}(x, t) \rightarrow f_{2}(x)$ uniformly on bounded subsets of $[B]$.

Proof. (i) It is sufficient to show that

$$
\left(\mathscr{F}_{1}^{-1} v_{t t}\right)(x, t)=\left(\mathscr{F}_{1}^{-1} v\right)_{t t}(x, t) .
$$

To see this, we let $w(y, t)=v_{t}(y, t)$ i.e.

$$
\begin{aligned}
w(y, t)= & -\sum_{n=1}^{\infty}\left(n^{k / 2} / n !\right)\left(\sin \left(n^{k / 2} t\right)\right) D^{n} g_{1}(0) y^{n} \\
& +\sum_{n=1}^{\infty}\left(\left(\cos \left(n^{k / 2} t\right)\right) / n !\right) D^{n} g_{2}(0) y^{n}
\end{aligned}
$$

It follows

$$
\begin{aligned}
\left|w_{t}(y, t)\right| \leqslant & (1 / 2 \pi) \int_{|\lambda|=2}\left(\sum_{n=1}^{\infty} n^{k / 2} / 2^{n+1}\right)\left|g_{1}(\lambda y)\right||d \lambda| \\
& +(1 / 2 \pi) \int_{|\lambda|=2}\left(\sum_{n=1}^{\infty} 1 / 2^{n+1}\right)\left|g_{2}(\lambda y)\right||d \lambda| .
\end{aligned}
$$

Employing arguments similar to the proof of Theorem 4.7(i), we see that condition (12) of Lemma 4.6 is satisfied by $w(y, t)$, and so $\mathscr{F}_{1}^{-1} w_{t}(x, t)=\left(\mathscr{F}_{1}^{-1} w\right)_{t}(x, t)$.

Applying the same argument once more, we obtain

$$
\left(\mathscr{F}_{1}^{-1} w\right)=\left(\mathscr{F}_{1}^{-1} v_{t}\right)=\left(\mathscr{F}_{1}^{-1} v\right)_{t}
$$

(7) follows immediately from the preceding two identities.

(ii) follows by the same techniques in the proof of Theorem 4.7(ii). 
Next, we define the semigroup associated with the problem $v_{t t}(y, t)=$ $-\tilde{\mathscr{T}}^{k} v(y, t) ; v(y, 0)=g_{1}(y)$ and $v_{t}(y, 0)=g_{2}(y)$ as follows. Let

$$
A_{n}(t)=\left[\begin{array}{ll}
P_{n}(t) & Q_{n}(t) \\
P_{n}^{\prime}(t) & Q_{n}^{\prime}(t)
\end{array}\right]
$$

Define for $t \geqslant 0$ a linear operator $T_{t}$ on $\tilde{E}_{a} \times \tilde{E}_{a}$ by

$$
T_{t}\left[\begin{array}{l}
g_{1} \\
g_{2}
\end{array}\right]=\sum_{n=0}^{\infty} \frac{1}{n !} A_{n}(t)\left[\begin{array}{l}
D^{n} g_{1}(0) x^{n} \\
D^{n} g_{2}(0) x^{n}
\end{array}\right] .
$$

Let $\left\|\left(g_{1}, g_{2}\right)\right\|_{N}=\left(\left\|g_{1}\right\|_{N}^{2}+\left\|g_{2}\right\|_{N}^{2}\right)^{1 / 2}, N=1,2,3, \ldots$ Then $\left\{\|(,)\|_{N}\right\}$ defines a sequence of norms on $\tilde{\mathcal{E}}_{a} \times \tilde{\mathcal{E}}_{a}$. With the topology induced by $\left\{\|(,)\|_{N}\right\}, \tilde{\varepsilon}_{a} \times \tilde{\mathcal{E}}_{a}$ obviously also forms a (sequentially) complete metrizable topological convex space (see 4.9).

Obviously,

$$
T_{t}\left[\begin{array}{l}
g_{1} \\
g_{2}
\end{array}\right] \in \tilde{\varepsilon}_{a} \times \tilde{\varepsilon}_{a}
$$

It follows by (16) of $\$ 4$ that

$$
\begin{aligned}
\left\|T_{t}\left[\begin{array}{l}
g_{1} \\
g_{2}
\end{array}\right]\right\|_{N} & \leqslant\left[\left(2\left\|g_{1}\right\|_{2 N}+2 t\left\|g_{2}\right\|_{2 N}\right)^{2}+\left(\left(\sum_{n=1}^{\infty} n^{k / 2} / 2^{n}\right)\left\|g_{1}\right\|_{2 N}+2\left\|g_{2}\right\|_{2 N}\right)^{2}\right]^{1 / 2} \\
& \leqslant 2\left(\max \left(2 t, \sum_{n=1}^{\infty}\left(n^{k / 2} / 2^{n}\right)\right)\right)\left\|\left(g_{1}, g_{2}\right)\right\|_{2 N}
\end{aligned}
$$

i.e., $T_{t}$ is continuous. Moreover, we have

Proposition 5.4. (i) $T_{t+s}=T_{t} T_{s}$ for $t, s \geqslant 0 ; T_{0}=I$.

(ii) $\lim _{t \rightarrow t_{0}} T_{t}\left[\begin{array}{l}g_{1} \\ g_{2}\end{array}\right]=T_{t_{0}}\left[\begin{array}{l}g_{1} \\ g_{2}\end{array}\right]$.

(iii) If $S$ is a compact set in $[0, \infty)$, then the family $\left\{T_{s}: s \in S\right\}$ of operators is equicontinuous. That is, given any continuous seminorm $q$ on $\tilde{\mathcal{E}}_{a} \times \tilde{\mathcal{E}}_{a}$, there is a constant $C_{1}$ and a seminorm $\|\cdot\|_{N}$ such that

$$
q\left(T_{t}\left[\begin{array}{l}
g_{1} \\
g_{2}
\end{array}\right]\right) \leqslant C_{1}\left\|\left[\begin{array}{l}
g_{1} \\
g_{2}
\end{array}\right]\right\|_{N}
$$

REMARK 2. $T_{t}$ is not an equicontinuous family of operators. To see this, let $g_{1}(x)=0, g_{2}(x)=1$ in (8). We obtain

$$
T_{t}\left[\begin{array}{l}
0 \\
1
\end{array}\right]=t \rightarrow \infty \quad \text { as } t \rightarrow \infty
$$

By standard computations, the following two propositions are obvious.

Proposition 5.5. Let

$$
A=\left[\begin{array}{cc}
0 & I \\
-\tilde{\vartheta}^{k} & 0
\end{array}\right]
$$

Then $A$ is the infinitesimal generator of $T_{t}$ and the domain of $A$ is $\tilde{\mathcal{E}}_{a} \times \tilde{\mathcal{E}}_{a}$. 
Proposition 5.6. Let $U_{t}=e^{-t} T_{t}, t \geqslant 0$. Then the family of operators $\left\{U_{t}\right\}$ forms an equicontinuous semigroup of class $\left(C_{0}\right)$ on $\tilde{\mathcal{E}}_{a} \times \tilde{\mathcal{E}}_{a}$ and its infinitesimal generator is given by $A-I$.

COROLlary 5.7. There is a unique solution $W(x, t)$ such that both $W(x, t)$ and $W_{t}(x, t)$ satisfy the conditions (i), (ii) of Theorem 4.14 and

$$
\begin{gathered}
W_{t t}(x, t)+2 W_{t}(x, t)=-\tilde{\Upsilon}^{k} W(x, t)-W(x, t), \\
W(x, 0)=g_{1}(x) ; W_{t}(x, 0)=g_{2}(x)-g_{1}(x)
\end{gathered}
$$

where $g_{1}, g_{2}$ are in $\tilde{E}_{a}$. Moreover, the solution is given by $W(x, t)=e^{-t} v(x, t)$ where $v(x, t)$ is the function given by (6) in Theorem 5.2.

Proof. Let $H_{1}=W, H_{2}=W_{t}+W ;(10)$ becomes

$$
\left[\begin{array}{l}
H_{1} \\
H_{2}
\end{array}\right]_{t}=(A-I)\left[\begin{array}{l}
H_{1} \\
H_{2}
\end{array}\right] \text { where } A=\left(\begin{array}{cc}
0 & I \\
-\tilde{\mathscr{T}}^{k} & 0
\end{array}\right) \text {. }
$$

By Proposition 5.6 and a technique similar to the proof of Theorem 4.14, uniqueness follows immediately.

Next, we show that if $v(x, t)$ is any solution for the Cauchy problem (i) of Theorem 5.2, then $W(x, t)=e^{-t} v(x, t)$ satisfies (10). Indeed,

$$
W_{t}=-e^{-t} v+e^{-t} v_{t}, \quad W_{t}=e^{-t} v-2 e^{-t} v_{t}+e^{-t} v_{t}
$$

hence $W_{t t}+2 W_{t}=-e^{-t} v+e^{-t}\left(-\tilde{\mathscr{T}}^{k} v\right)=-\tilde{\mathfrak{N}}^{k} W-W$. Also,

$$
W_{t}(x, 0)=-v(x, 0)+v_{t}(x, 0)=g_{2}(x)-g_{1}(x)
$$

and

$$
W(x, 0)=v(x, 0)=g_{1}(x)
$$

COROLlary 5.8. The Cauchy problem (i) of Theorem 5.2 has a unique solution $v(x, t)$ such that $v(x, t)$ and $v_{t}(x, t)$ satisfy the conditions (i), (ii) of Theorem 4.14.

Proof. Let $v(x, t)$ be the solution given by (6) and $v_{1}(x, t)$ be any solution. Then as we have seen in the proof of Corollary 5.7, $W(x, t)=e^{-t} v_{1}(x, t)$ must be a solution of (10). By the hypotheses on $v_{1}, W(x, t)$ satisfies the hypotheses in Corollary 5.7. It follows by the uniqueness, $e^{-t} v(x, t)=e^{-t} v_{1}(x, t)$ for $t>0$; thus $v=v_{1}$.

Finally, we conclude that

Corollary 5.9. Assume $f_{1}, f_{2}$ are in $\mathcal{E}_{a}$. Then the Cauchy problem (2) has a unique solution $u(x, t)$ such that $u(x, t)$ and $u_{t}(x, t)$ satisfy the conditions (i), (ii) of Corollary 4.15 .

Proof. The uniqueness follows by taking the Fourier-Wiener 1-transform of (2) and by Corollary 5.8.

As an implication of Proposition 5.6, we have 
Corollary 5.10. For each pair $g_{1}, g_{2} \in \tilde{E}_{a}$, we have

$$
\frac{\partial}{\partial t} T_{t}\left[\begin{array}{l}
g_{1} \\
g_{2}
\end{array}\right]=A T_{t}\left[\begin{array}{l}
g_{1} \\
g_{2}
\end{array}\right]=T_{t} A\left[\begin{array}{l}
g_{1} \\
g_{2}
\end{array}\right] \text {. }
$$

Proof. It follows from Proposition 5.6 and [20, Chapter 9, Theorem 2] that

Since $T_{t}=e^{t} U_{t}$, we get

$$
\frac{\partial}{\partial t} U_{t}\left[\begin{array}{l}
g_{1} \\
g_{2}
\end{array}\right]=(A-I) U_{t}\left[\begin{array}{l}
g_{1} \\
g_{2}
\end{array}\right]=U_{t}(A-I)\left[\begin{array}{l}
g_{1} \\
g_{2}
\end{array}\right] \text {. }
$$

$$
\begin{aligned}
\frac{\partial}{\partial t} T_{t}\left[\begin{array}{l}
g_{1} \\
g_{2}
\end{array}\right] & =\left(e^{t} U_{t}+e^{t}(A-I) U_{t}\right)\left[\begin{array}{l}
g_{1} \\
g_{2}
\end{array}\right] \\
& =\left(e^{t} U_{t}+e^{t} U_{t}(A-I)\right)\left[\begin{array}{l}
g_{1} \\
g_{2}
\end{array}\right] \\
& =A T_{t}\left[\begin{array}{l}
g_{1} \\
g_{2}
\end{array}\right]=T_{t} A\left[\begin{array}{l}
g_{1} \\
g_{2}
\end{array}\right] \cdot \square
\end{aligned}
$$

As a further application of Theorems 5.2, 5.3 and Corollaries 5.8, 5.9, 5.10, we may consider the following Cauchy problem associated with (2).

$$
\begin{aligned}
& u_{t t}(x, t)=-\mathscr{T}^{k} u(x, t)+h(x, t) \quad \text { with } u(x, 0)=f_{1}(x), \\
& u_{t}(x, 0)=f_{2}(x), \quad f_{1}, f_{2} \in \mathcal{E}_{a} .
\end{aligned}
$$

Analogous to Theorem 4.16, we have

THEOREM 5.11. Assuming that $h(x, t)$ satisfies the three conditions (i)-(iii) in Theorem 4.16, then there is a unique solution $u(x, t)$ for the Cauchy problem (12) so that $u(x, t)$ and $u_{t}(x, t)$ satisfy the conditions (i), (ii) of Corollary 4.15.

Proof. Reduction. We may assume $f_{1}=0$ by replacing $u$ by $u-f_{1}$. Then the Cauchy problem becomes $u_{t t}(x, t)=-\mathscr{T}^{k} u(x, t)+h(x, t)-\mathscr{\vartheta}^{k} f_{1}(x)$ with $u(x, 0)$ $=0, u_{t}(x, 0)=f_{2}$. (Note that the three conditions (i)-(iii) remain valid for $h(x, t)$ $-\Re^{k} f_{1}(x)$, by Remark 5 of $\S 4$.)

We see that (12) is now equivalent to the Cauchy problem below

$$
\left[\begin{array}{l}
v_{1} \\
v_{2}
\end{array}\right]_{t}=\left[\begin{array}{cc}
0 & I \\
-\tau^{k} & 0
\end{array}\right]\left[\begin{array}{l}
v_{1} \\
v_{2}
\end{array}\right]+\left[\begin{array}{c}
0 \\
h(x, t)
\end{array}\right] ; \quad\left[\begin{array}{l}
v_{1} \\
v_{2}
\end{array}\right]_{t=0}=\left[\begin{array}{l}
0 \\
f_{2}
\end{array}\right]
$$

with $v_{1}=u, v_{2}=u_{t}$.

Imitating the proof of Theorem 4.16, we find that

$$
\left[\begin{array}{l}
v_{1} \\
v_{2}
\end{array}\right]=\mathscr{F}_{1}^{-1} T_{t} \mathscr{F}_{1}\left[\begin{array}{l}
0 \\
f_{2}
\end{array}\right]+\int_{0}^{t} \mathscr{F}_{1}^{-1} T_{t-s} \mathscr{F}_{1}\left[\begin{array}{c}
0 \\
h(x, t)
\end{array}\right] d s
$$

is the unique solution of (13). It follows that the solution of (12) (with $f_{1}=0$ ) is given by

$$
\begin{aligned}
u(x, t)= & \mathscr{F}_{1}^{-1}\left(\sum_{n=0}^{\infty}\left(Q_{n}(t) / n !\right) D^{n} \tilde{f}_{2}(0) y^{n}\right)(x) \\
& +\int_{0}^{t} \mathscr{F}_{1}^{-1}\left(\sum_{n=0}^{\infty}\left(Q_{n}(t-s) / n !\right) D^{n} \tilde{h}(0, s) y^{n}\right)(x) d s
\end{aligned}
$$


where " "" is the Fourier-Wiener 1-transform.

REMARK 3. In the next paper we shall continue to apply the Fourier-Wiener transform to investigate the existence and regularity of solutions of the following two types of differential equations:

(i) $u_{t}(x, t)=(-1)^{k+1} \Delta^{k} u(x, t) ; u(x, 0)=f(x)$.

(ii) $u_{t t}(x, t)=(-1)^{k+1} \Delta^{k} u(x, t) ; u(x, 0)=f(x), u_{t}(x, 0)=g(x)$.

\section{REFERENCES}

1. R. H. Cameron and W. T. Martin, Fourier-Wiener transforms of analytic functionals, Duke Math. J. 12 (1945), 489-507.

2. , Fourier-Wiener transforms of functionals belonging to $L_{2}$ over the space $\mathcal{C}$, Duke Math. J. 14 (1946), 99-107.

3. L. Gross, Integration and nonlinear transformations in Hilbert space, Trans. Amer. Math. Soc. 94 (1960), 404-440.

4. Harmonic analysis on Hilbert space, Mem. Amer. Math. Soc., No. 46, 1963.

5. Abstract Wiener spaces, Proc. Fifth Berkeley Sympos. Math. Stat. and Prob. (Berkeley, Calif., 1965/66), Vol. II: Contributions to Probability Theory, Part I, Univ. California Press, Berkeley, Calif., 1967, pp. 31-42.

6. $ـ$ Potential theory on Hilbert space, J. Functional Analysis 1 (1967), 123-181.

7. T. Hida, Stationary stochastic processes, Princeton Univ. Press, Princeton, N. J., 1970.

8. E. Hille, Functional analysis and semi-groups, Amer. Math. Soc. Colloq. Publ., vol. 31, Amer. Math. Soc., Providence, R. I., 1948.

9. E. Hille and R. S. Phillips, Functional analysis and semi-groups, rev. ed., Amer. Math. Soc. Colloq. Publ., vol. 31, Amer. Math. Soc., Providence, R. I.; reprinted 1974.

10. H.-H. Kuo, Integration by parts for abstract Wiener measures, Duke Math. J. 41 (1974), 373-379.

11. __ Gaussian measures in Banach spaces, Lecture Notes in Math., vol. 463, Springer-Verlag, Berlin and New York, 1975.

12. E. Nelson, Dynamical theories of Brownian motion, Princeton Univ. Press, Princeton, N. J., 1967.

13. M. A. Piech, A fundamental solution of the parabolic equation on Hilbert space, J. Functional Analysis 3 (1969), 85-114.

14. _ Parabolic equations associated with the number operator, Trans. Amer. Math. Soc. 194 (1974), 213-222.

15. The Ornstein-Uhlenbeck semi-group in an infinite dimensional $L^{2}$ setting, J. Functional Analysis 18 (1975), 271-285.

16. I. E. Segal, Tensor algebra over Hilbert space. I, Trans. Amer. Math. Soc. 81 (1956), 106-134.

17. _ Distributions in Hilbert space and canonical systems of operators, Trans. Amer. Math. Soc. 88 (1958), 12-41.

18. A. V. Skorohod, Integration in Hilbert space, Springer-Verlag, Berlin and New York, 1974.

19. A. V. Uglanov, Differential equations with constant coefficients for generalized measures on Hilbert space, Math. USSR Izv. 9 (1975), 414-440.

20. K. Yosida, Functional analysis, rev. ed., Springer-Verlag, Berlin and New York, 1970.

Department of Mathematics, State University of New York at Buffalo, Buffalo, New York 14214

Current address: Department of Mathematics, National Cheng-Kung University, Tainan, Taiwan 700 